The Astrophysical Journal, 601:512-529, 2004 January 20

(C) 2004. The American Astronomical Society. All rights reserved. Printed in U.S.A.

\title{
SIMULATIONS OF CORE CONVECTION IN ROTATING A-TYPE STARS: DIFFERENTIAL ROTATION AND OVERSHOOTING
}

\author{
Matthew K. Browning, Allan Sacha Brun, ${ }^{1}$ and Juri Toomre \\ JILA and Department of Astrophysical and Planetary Sciences, Campus Box 440, University of Colorado, Boulder, CO 80309-0440 \\ Received 2003 August 13; accepted 2003 September 25
}

\begin{abstract}
We present the results of three-dimensional simulations of core convection within A-type stars of $2 M_{\odot}$, at a range of rotation rates. We consider the inner $30 \%$ by radius of such stars, thereby encompassing the convective core and some of the surrounding radiative envelope. We utilize our anelastic spherical harmonic code, which solves the compressible Navier-Stokes equations in the anelastic approximation, to examine highly nonlinear flows that can span multiple scale heights. The cores of these stars are found to rotate differentially, with central cylindrical regions of strikingly slow rotation achieved in our simulations of stars whose convective Rossby number $\left(R_{o c}\right)$ is less than unity. Such differential rotation results from the redistribution of angular momentum by the nonlinear convection that strongly senses the overall rotation of the star. Penetrative convective motions extend into the overlying radiative zone, yielding a prolate shape (aligned with the rotation axis) to the central region in which nearly adiabatic stratification is achieved. This is further surrounded by a region of overshooting motions, the extent of which is greater at the equator than at the poles, yielding an overall spherical shape to the domain experiencing at least some convective mixing. We assess the overshooting achieved as the stability of the radiative exterior is varied and the weak circulations that result in that exterior. The convective plumes serve to excite gravity waves in the radiative envelope, ranging from localized ripples of many scales to some remarkable global resonances.
\end{abstract}

Subject headings: convection — stars: interiors — stars: rotation

\section{INTRODUCTION AND MOTIVATION}

Convection within the cores of massive stars has major impact on their structure and evolution, yet little is known about the detailed properties of such convection. These convective motions, driven by the steep temperature gradient arising from the vigorous burning of the CNO cycle, carry outward a large fraction of the stars' luminosities. In standard one-dimensional stellar models (e.g., Maeder \& Meynet 2000), the effects of such convection are usually calculated using simple mixing-length prescriptions, but such approaches involve considerable uncertainties. Mixing-length theory can provide only rough estimates of the energy flux carried by the convection and no effective estimates of the differential rotation or meridional circulation generated by the convective flows, which may have important consequences for mixing and for redistribution of angular momentum within massive stars.

Overshooting from convection zones raises other problems. It has long been realized that convective motions are unlikely to come to a halt at the boundary between the convective core and the stable radiative zone above it. Indeed, upward moving fluid parcels will penetrate into the stable zone, decelerating and mixing with their surroundings (e.g., Roxburgh 1965). Such overshooting motions might bring fresh fuel into the core, thereby prolonging a star's lifetime on the main sequence, and have noticeable effects on its evolution (e.g., Woo \& Demarque 2001). Yet estimating the extent of this overshooting is challenging.

Likewise uncertain is the differential rotation achieved within convective cores. In the solar convection zone, Reynolds

\footnotetext{
${ }^{1}$ New permanent address: DSM/DAPNIA/Service d'Astrophysique, CEA Saclay, 91191 Gif-sur-Yvette, France.
}

stresses, meridional circulations, and viscous forces give rise to a prominent differential rotation, now being probed extensively by helioseismology (Gough \& Toomre 1991; Schou et al. 1998). Explaining that differential rotation within the Sun has been a major challenge for theory and simulation in recent years, with three-dimensional spherical shell simulations of turbulent convection now beginning to make contact with the helioseismic results (e.g., Miesch et al. 2000; Elliott, Miesch, \& Toomre 2000; Brun \& Toomre 2002). The presence of such nonuniform rotation deep within more massive stars would have major consequences for the properties of such stars: differential rotation can give rise to shear instabilities that stir and mix material, and may serve to build strong magnetic fields through sustained dynamo action.

Indeed, the generation of magnetic fields within stars by dynamo action must result from large-scale convection in the stellar plasma interacting with rotation. In some stars with convective envelopes, such as the Sun, the building of orderly magnetic fields and cyclic activity is thought to depend sensitively on highly turbulent convection yielding strong differential rotation, including a tachocline of shear at the interface between the bottom of the convection zone and the radiative interior (e.g., Thompson et al. 2003). More generally, correlations between magnetic fields and rotation have been inferred in main-sequence stars ranging from F5 to M9 (Noyes et al. 1984; Mohanty et al. 2002), with these fields also thought to be a result of dynamo action driven by convection, whether occupying an envelope or the full interior. Yet a comprehensive understanding of how this occurs remains elusive. More massive stars with convective cores are similarly likely to admit magnetic dynamo action, for they too possess the necessary ingredients: intensely turbulent convection, a highly conductive medium, and global effects of rotation, all thought to be 
crucial for the building of magnetic fields (Charbonneau \& MacGregor 2001). Whether the resulting fields are chaotic or global and orderly may be a sensitive matter.

\subsection{Estimates of Overshooting from Convective Cores}

Some theoretical work has provided constraints on the size of the convective core and the extent of overshooting. Roxburgh (1978, 1989, 1998; see also Zahn 1991) showed that an upper limit to the total size of the convective region can be deduced by considering the integral

$$
\int_{0}^{r_{c}}\left(L_{\mathrm{rad}}-L\right) \frac{1}{T^{2}} \frac{d T}{d r} d r=\int_{V} \frac{\Phi}{T} d V>0,
$$

with $L$ the total nuclear luminosity, $L_{\text {rad }}$ the radiative luminosity, $\Phi$ the viscous dissipation per unit volume, and $r_{c}$ the radius of the convective core including overshooting. Since within the convective core some of the nuclear energy must be carried by convection, $L_{\text {rad }}<L$ there. Thus, if viscous dissipation is neglected, there must be an overshooting region where $L_{\mathrm{rad}}>L$, whose extent can be estimated from this equation. For small convective cores, these considerations yield an overshoot region of size $d \approx 0.18 r_{o}$, where $r_{o}$ is the radius of the convectively unstable region (Roxburgh 1992). Rosvick \& VandenBerg (1998) found that observations of the stellar cluster NGC 6819 were best fitted by overshooting that is about half the upper limit provided by Roxburgh's constraint.

Various attempts have been made to estimate the extent of overshooting from observations, typically expressed in terms of pressure scale heights. Meynet, Mermilliod, \& Maeder (1993), for example, found that best-fit isochrones for a large number of clusters required overshooting that extends for about $0.2-0.3$ times the pressure scale height. Perryman et al. (1998) found evidence for a similar degree of overshooting in Hyades stars. In the open cluster M67, whose main-sequence turnoff stars are thought to be near the low-mass end of stars possessing convective cores, some authors have suggested overshooting that is of order 0.1 pressure scale heights (e.g., Maeder \& Meynet 1991; Carraro et al. 1994).

\subsection{Challenges Raised by A-Type Stars}

Convective cores are realized for main-sequence stars more massive than about $1.2 M_{\odot}$, thereby providing many stars in which the effects of overshooting could be assessed. However, A-type stars exhibit a variety of peculiarities that have made them the subject of particularly detailed study (see Wolff 1983, hereafter W83, for a broad review). We recall that A-type stars possess both a convective core as well as multiple shallow convection zones near the surface. Some of these stars display strong abundance anomalies, with greatly enhanced rare earth element abundances relative to normal stars (e.g., Kurtz 1990). Surface abundance anomalies have also been observed in a variety of other stars (Preston 1974; Gehren 1988; Pinsonneault 1997; Abt 2000). Radiative diffusive separation, wherein radiation pressure drives outward some elements while others sink, is a favored explanation for these abundance features but requires a very stable radiative zone in which this may be occurring. Latour, Toomre, \& Zahn (1981) showed that the H and $\mathrm{He}$ envelope convection zones in A-type stars are likely linked by penetrative motions that would upset the delicate quiescence needed for radiative diffusion to work effectively. This problem can be avoided if helium gravitationally settles out to greater depths, in which case the deeper convection zone driven primarily by that element's second ionization would not exist (e.g., Vauclair, Vauclair, \& Pamjatnikh 1974). Such settling is impeded by meridional circulations within the radiative zone (Michaud et al. 1983), which are thought to increase in amplitude with increasing stellar rotational velocity. It has recently been shown (e.g., Richard, Michaud, \& Richer 2001) that in some stars a further iron convection zone is established below the other two surface convective regions, which may lead to deeper mixing within such stars (e.g., Vauclair 2003; Richer, Michaud, \& Turcotte 2000). While this basic picture explains many of the observed abundance features, many of the details are not precisely known.

A subset of the A-type stars also possesses strong surface magnetic features that appear to persist for many rotation periods (W83). Such fields may well be oblique rotators of primordial origin (e.g., Mestel 1999). However, if dynamogenerated fields within the convective core were able to rise through the radiative zone, possibly by means of magnetic buoyancy instabilities, they might also influence the surface fields. Recent numerical studies (MacGregor \& Cassinelli 2003) have provided tantalizing indications that it may indeed be possible for strong magnetic fields generated in the core to rise to the surface.

The A-type stars also hold interest because of the rich set of observational constraints beginning to be provided by asteroseismological probing of such stars. The pulsating Ap stars exhibit high-order nonradial $p$-modes, which allow some deductions about stellar radii, temperature, and magnetic fields (e.g., Matthews 1991; Cunha 2002).

\subsection{Modeling Convection in Full Spherical Domains}

The extensive observations of A-type stars and the challenges raised have encouraged us to consider the core convection influenced by rotation that is occurring deep within their interiors. The surface pathologies of A stars clearly raise many questions about their interior dynamics, thereby lending vibrancy to their study. We hope that our modeling will further serve to reveal general dynamical properties of the core convection that is also occurring in other massive stars.

The major uncertainties associated with core convectionthe extent of its overshooting into the surrounding radiative envelope and the differential rotation and circulations it establishes - now lead us to undertake simulations of such convection in full spherical geometries that permit global connectivity. We aim to capture much of the essential physics, admitting highly nonlinear flows that can extend over multiple scale heights as they mold the dynamical structure of the convective core. We realize that there may be few observable consequences at the surfaces of these stars of what may be proceeding dynamically at their centers. However, the circulations and gravity waves that may be induced by the core convection may well have implications at the surface, as might magnetic fields being produced by dynamo action deep within these stars. All may depend somewhat sensitively on the rotation rates of the stars.

Advances in supercomputing are now beginning to allow us to examine the properties of core convection in some detail. We begin here by considering three-dimensional hydrodynamic simulations of the inner regions of a $2 M_{\odot}$ A-type star. In subsequent papers, we plan to explore the magnetic dynamo action that may be realized in such convective cores and to examine possible instabilities that might allow the resulting magnetic fields to rise to the surface. 
In $\S 2$ we describe our formulation of the problem and briefly summarize the computational tools being used. Section 3 discusses the general properties of the nonlinear convective flows realized in the core, including the transport of energy achieved. The redistribution of angular momentum by the convection yields prominent differential rotation profiles that are presented in $\S 4$. Section 5 considers the temporal evolution of convective patterns within the core and examines the gravity waves that are excited within the radiative envelope. Analysis of the penetration and overshooting by the convection is discussed in $\S 6$. The meridional circulations induced by the convection, within both the core and the radiative envelope, are considered in $\S 7$. Section 8 evaluates the manner in which the convection yields strong differential rotation. A summary of our principal findings and their implications is presented in $\S 9$.

\section{POSING THE PROBLEM}

\subsection{Anelastic Equations}

The simulations described here were performed using our anelastic spherical harmonic (ASH) code. ASH solves the three-dimensional anelastic equations of motion in a rotating spherical geometry using a pseudospectral semi-implicit approach (Clune et al. 1999; Miesch et al. 2000). These equations are fully nonlinear in velocity variables and linearized in thermodynamic variables with respect to a spherically symmetric mean state. This mean state is taken to have density $\bar{\rho}$, pressure $\bar{P}$, temperature $\bar{T}$, and specific entropy $\bar{S}$; perturbations about this mean state are written as $\rho, P, T$, and $S$, respectively. The conservation equations of mass, momentum, and energy in this rotating reference frame are therefore written as

$$
\begin{gathered}
\nabla \cdot(\bar{\rho} \boldsymbol{v})=0 \\
\bar{\rho}\left[\frac{\partial \boldsymbol{v}}{\partial t}+(\boldsymbol{v} \cdot \nabla) \boldsymbol{v}+2 \Omega_{0} \times \boldsymbol{v}\right]=-\nabla \boldsymbol{P}+\rho \boldsymbol{g}-\nabla \cdot \mathbf{D} \\
-(\nabla \bar{P}-\bar{\rho} \boldsymbol{g}), \\
\bar{\rho} \bar{T} \frac{\partial S}{\partial t}=\nabla \cdot\left[\kappa_{r} \bar{\rho} c_{p} \nabla(\bar{T}+T)+\kappa \bar{\rho} \bar{T} \nabla(\bar{S}+S)\right] \\
-\bar{\rho} \bar{T} \boldsymbol{v} \cdot \nabla(\bar{S}+S)+2 \bar{\rho} \nu\left[\mathbf{e}_{i j} \mathbf{e}_{i j}-\frac{1}{3}(\nabla \cdot \boldsymbol{v})^{2}\right]+\bar{\rho} \epsilon,
\end{gathered}
$$

where $c_{p}$ is the specific heat at constant pressure, $\boldsymbol{v}=\left(v_{r}, v_{\theta}\right.$, $\left.v_{\phi}\right)$ is the local velocity in spherical geometry in the rotating frame of constant angular velocity $\Omega_{0}, \boldsymbol{g}$ is the gravitational acceleration, $\kappa_{r}$ is the radiative diffusivity, $\epsilon$ is the heating rate per unit mass due to nuclear energy generation, and $\mathbf{D}$ is the viscous stress tensor, with components

$$
\mathbf{D}_{i j}=-2 \bar{\rho} \nu\left[\mathbf{e}_{i j}-\frac{1}{3}(\nabla \cdot \boldsymbol{v}) \delta_{i j}\right],
$$

where $\mathbf{e}_{i j}$ is the strain rate tensor. Here $\nu$ and $\kappa$ are effective eddy diffusivities for vorticity and entropy. To close the set of equations, linearized relations for the thermodynamic fluctuations are taken as

$$
\frac{\rho}{\bar{\rho}}=\frac{P}{\bar{P}}-\frac{T}{\bar{T}}=\frac{P}{\gamma \bar{P}}-\frac{S}{c_{p}},
$$

assuming the ideal gas law

$$
\bar{P}=\mathcal{R} \bar{\rho} \bar{T},
$$

where $\mathcal{R}$ is the gas constant. The effects of compressibility on the convection are taken into account by means of the anelastic approximation, which filters out sound waves that would otherwise severely limit the time steps allowed by the simulation.

Convection in stellar environments occurs over a large range of scales. Numerical simulations cannot, with present computing technology, consider all these scales simultaneously. We therefore seek to resolve the largest scales of the nonlinear flow, which we think are likely to be the dominant players in establishing differential rotation and other mean properties of the core convection zone. We do so within a large-eddy simulation formulation, which explicitly follows larger scale flows while employing subgrid-scale descriptions for the effects of the unresolved motions. Here those unresolved motions are treated as enhancements to the viscosity and thermal diffusivity ( $\nu$ and $\kappa$ ), which are thus effective eddy viscosities and diffusivities. For simplicity, we have taken these to be functions of radius alone and to scale as the inverse of the square root of the mean density. We emphasize that currently tractable simulations are still many orders of magnitude away in parameter space from the highly turbulent conditions likely to be found in real stellar convection zones. These large-eddy simulations should therefore be viewed only as indicators of the properties of the real flows. We are encouraged, however, by the success that similar simulations (e.g., Miesch et al. 2000; Elliott et al. 2000; Brun \& Toomre 2002) have enjoyed in matching the detailed observational constraints for the differential rotation within the solar convection zone provided by helioseismology.

\subsection{Computational Approach}

Thermodynamic variables within ASH are expanded in spherical harmonics $Y_{\ell}^{m}(\theta, \phi)$ in the horizontal directions and in Chebyshev polynomials $T_{n}(r)$ in the radial. Spatial resolution is thus uniform everywhere on a sphere when a complete set of spherical harmonics of degree $\ell$ is used, retaining all azimuthal orders $m$. We truncate our expansion at degree $\ell=\ell_{\max }$, which is related to the number of latitudinal mesh points $N_{\theta}$ [here $\left.\ell_{\max }=\left(2 N_{\theta}-1\right) / 3\right]$, take $N_{\phi}=2 N_{\theta}$ latitudinal mesh points, and utilize $N_{r}$ collocation points for the projection onto the Chebyshev polynomials. We employ a stacked Chebyshev representation, wherein the computational domain is split into two regions and separate Chebyshev expansions performed for each. The interface between these two regions, here taken to be the approximate boundary between the convective and radiative zones, is thus treated with greater resolution in order to capture elements of the penetrative convection occurring there. We have taken $N_{r}=49+33=82$ and $\ell_{\max }=85$ in this study for the cases considered here and have used $\ell_{\max }=170$ for some companion simulations to verify that the spectral resolution was adequate. An implicit second-order CrankNicholson scheme is used in determining the time evolution of the linear terms, whereas an explicit second-order AdamsBashforth scheme is employed for the advective and Coriolis terms. The ASH code has been optimized to run efficiently on massively parallel supercomputers such as the IBM SP-3 and the Compaq TCS-1 and has demonstrated excellent scalability on such machines. 


\subsection{Formulation of the Model}

The model considered here is intended to be a simplified description of the inner $30 \%$ by radius of a main-sequence A-type star of $2 M_{\odot}$, consisting of the convective core (approximately the inner $15 \%$ of the star) and a portion of the overlying radiative zone. Contact is made with a one-dimensional stellar model (at an age of $500 \mathrm{Myr}$ ) for the initial conditions, adopting realistic values for the radiative opacity, density, and temperature. This one-dimensional model was obtained using the CESAM stellar evolution code (Morel 1997; Brun et al. 2002), which employs the OPAL equation of state and opacities (Iglesias \& Rogers 1996; Rogers, Swenson, \& Iglesias 1996) and the nuclear cross sections of Adelberger et al. (1998) in describing the microscopic properties of the stellar plasma. Convection is computed within CESAM using a classical mixing-length treatment calibrated on solar models, with some convective overshooting (typically $r=0.25 H_{p}$ ) taken into account. Our simulations were initialized using the radial profiles of gravity $g$, radiative diffusivity $\kappa_{\text {rad }}$, mean density $\bar{\rho}$, and mean entropy gradient $d \bar{S} / d r$ as the starting points for an iterative Newton-Raphson solution for the hydrostatic balance and for the gradients of the thermodynamic variables. The mean temperature $\bar{T}$ is then deduced from equation (7). This technique yields mean profiles in reasonable agreement with the one-dimensional stellar model (Fig. 1). The nuclear source of energy $\epsilon$ in our simulations is also deduced from the stellar model. For computational convenience, we take $\epsilon=$ $\epsilon_{0} \bar{T}^{8}$, determining the constant $\epsilon_{0}$ by requiring that the integrated luminosity match the one-dimensional model's surface luminosity.

We have softened the steep entropy gradient contrast encountered in going from the convective core to the surrounding radiative zone, which would otherwise favor the driving of small-scale, high-frequency internal gravity waves that are presently unresolvable given reasonable computational resources. This lessening of the "stiffness" of the system has impact on the extent to which convective motions may overshoot into the radiative region. We have excluded the inner $2 \%$

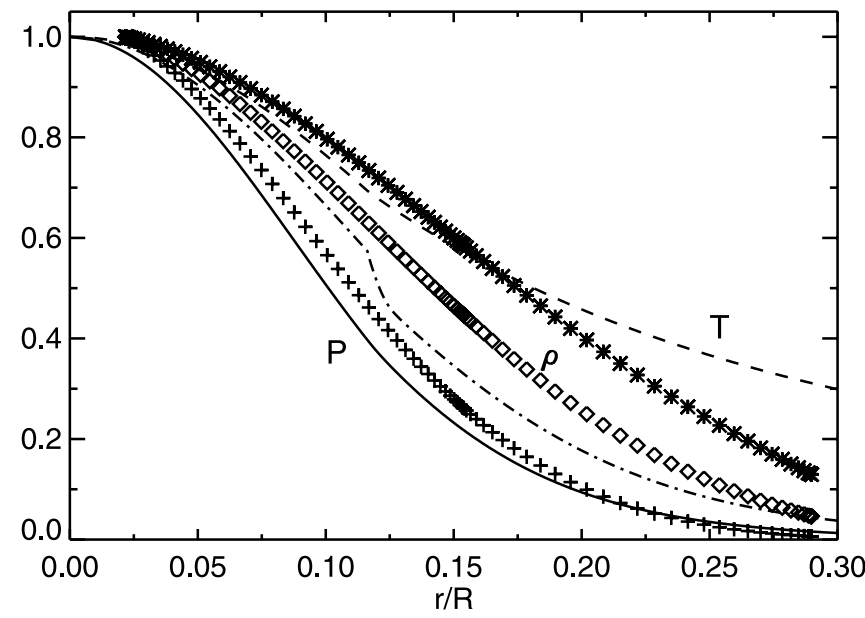

FIG. 1.-Mean radial stratification in the central regions of a $2 M_{\odot}$ A-type star, within both a one-dimensional stellar structure model and case $\mathrm{C}$. The mean pressure $\bar{P}$, temperature $\bar{T}$, and density $\bar{\rho}$ are plotted with symbols at their radial mesh locations for case $\mathrm{C}$ and as continuous curves for the onedimensional model. All variables are normalized to their maximum values, which for case $\mathrm{C}$ at the inner boundary of the computational domain $(r=$ $0.022 R)$ are $\bar{\rho}_{0}=48 \mathrm{~g} \mathrm{~cm}^{-3}, \bar{T}_{0}=2.0 \times 10^{7} \mathrm{~K}$, and $\bar{P}_{0}=1.4 \times 10^{17}$ dynes $\mathrm{cm}^{-2}$. of the star from our computational domain for numerical reasons, namely, that the coordinate systems employed in ASH possess both coordinate singularities at $r=0$ and decreasing mesh size (and hence highly limited time steps) with decreasing radius. It is difficult to gauge with certainty the effects of excluding this central region, which might in principle both project a Taylor column aligned with the rotation axis (e.g., Pedlosky 1987) into the surrounding fluid, as well as give rise to boundary layers. However, we have seen no evidence that spurious physical responses have been generated by its omission. In trial simulations with both smaller and larger excluded central regions (ranging from $0.3 \%$ to $4 \%$ of the stellar radius), the properties of the developed mean flows were very similar to those described here.

As boundary conditions on the spherical domain, we utilize impenetrable and stress-free conditions for the velocity field and constant entropy gradient (i.e., constant emergent flux) at both the inner and outer boundaries. As initial conditions, some simulations have been started from quiescent conditions of uniform rotation whose radial stratification is given by the onedimensional stellar model; others used evolved states from prior solutions to initiate simulations with reduced diffusivities.

The main parameters of our simulations are summarized in Table 1. In brief, we have modeled the inner regions of $2 M_{\odot}$ A-type stars at a range of rotation rates, with nonlinear flows of varying complexity achieved by modifying the effective eddy viscosities and diffusivities $\nu$ and $\kappa$. Several values of $\nu$ and $\kappa$ are utilized to achieve both laminar and more complex convective flows in some companion cases, all at a Prandtl number $P_{r}=\nu / \kappa=0.25$. We consider rotation rates from $1 / 10$ to 4 times the solar rate of $\Omega_{0}=2.6 \times 10^{-6} \mathrm{~s}^{-1}$ (or $414 \mathrm{nHz}$ ), though concentrating primarily on models at the solar rotation rate. Three different values of the maximum entropy gradient $d \bar{S} / d r$ in the radiative region were taken, thus effectively varying the stiffness of the boundary between that region and the convective core, primarily as a way to study the effects on overshooting motions induced by softening that boundary. We have chosen only representative simulations for summary in this paper (and in Table 1) out of a larger total set of computations.

Figure 1 shows the mean radial profiles for temperature, density, and pressure attained in a representative simulation (case C), together with profiles of the same variables in a typical one-dimensional stellar structure model. The evolved simulation possesses somewhat less steep gradients for these variables than does the one-dimensional stellar model, but the functional form of these quantities is largely unchanged by our detailed treatment of the convection.

\section{NATURE OF CORE CONVECTION}

The flows in all the cases studied are highly time dependent, with complex and intermittent features emerging as the simulations evolve. The convection is characterized by large-scale flows that sweep through much of the unstable core, coupling widely separated sites. Thus, influences of the convective motions are decidedly nonlocal. Such global motions can result because of the topologically connected nature of full spheres. The addition of rotation to these spherical domains admits Coriolis forces and resulting velocity correlations (Reynolds stresses) that yield some surprising effects. In particular, we find that the cores exhibit prominent differential rotation in both radius and latitude, involving in most of our simulations a central column of slow rotation aligned with the rotation axis. 
TABLE 1

Summary of Simulation Parameters

\begin{tabular}{|c|c|c|c|c|c|c|c|c|c|c|c|c|c|c|}
\hline Parameter & & A & & $\mathrm{B}$ & & $\mathrm{C}$ & & $\mathrm{D}$ & & $\mathrm{E}$ & & $\mathrm{C} 4$ & & $\mathrm{~F}$ \\
\hline$\Omega_{0} / \Omega_{\odot} \ldots \ldots \ldots \ldots \ldots \ldots \ldots \ldots$ & 1 & & 1 & & 1 & & 1 & & 1 & & 4 & & 0.1 & \\
\hline $\max d \bar{S} / d r$ & 1.5 & $\times 10^{-6}$ & 1.5 & $\times 10^{-5}$ & 1.5 & $\times 10^{-5}$ & 1.5 & $\times 10^{-4}$ & 1.5 & $\times 10^{-4}$ & 1.5 & $\times 10^{-5}$ & 1.5 & $\times 10^{-4}$ \\
\hline & 4.35 & $\times 10^{11}$ & 2.49 & $\times 10^{12}$ & 4.35 & $\times 10^{11}$ & 2.49 & $\times 10^{12}$ & 4.35 & $\times 10^{11}$ & 2.49 & $\times 10^{11}$ & 4.35 & $\times 10^{11}$ \\
\hline & 1.74 & $\times 10^{12}$ & 9.96 & $\times 10^{12}$ & 1.74 & $\times 10^{12}$ & 9.96 & $\times 10^{12}$ & 1.74 & $\times 10^{12}$ & 9.96 & $\times 10^{11}$ & 1.74 & $\times 10^{12}$ \\
\hline$R_{a}$ & 1.7 & $\times 10^{5}$ & 2.0 & $\times 10^{4}$ & 1.9 & $\times 10^{5}$ & 1.0 & $\times 10^{4}$ & 3.3 & $\times 10^{5}$ & 2.2 & $\times 10^{6}$ & 3.3 & $\times 10^{5}$ \\
\hline$T_{a}$ & 1.2 & $\times 10^{7}$ & 3.6 & $\times 10^{5}$ & 1.2 & $\times 10^{7}$ & 3.6 & $\times 10^{5}$ & 1.2 & $\times 10^{7}$ & 5.9 & $\times 10^{8}$ & 1.2 & $\times 10^{5}$ \\
\hline 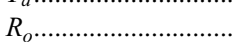 & 0.24 & & 0.47 & & 0.25 & & 0.33 & & 0.33 & & 0.12 & & 3.3 & \\
\hline 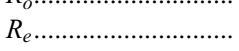 & 1.7 & $\times 10^{2}$ & 1.2 & $\times 10^{1}$ & 1.3 & $\times 10^{2}$ & 1.1 & $\times 10^{1}$ & 1.4 & $\times 10^{2}$ & 2.0 & $\times 10^{2}$ & 1.7 & $\times 10^{2}$ \\
\hline
\end{tabular}

Notes.-All simulations have an inner radius of $3.0 \times 10^{9} \mathrm{~cm}$ and an outer radius of $4.0 \times 10^{10} \mathrm{~cm}$, with $L=1.7 \times 10^{10} \mathrm{~cm}$ the approximate radial extent of the convective core. The Prandtl number $P_{r}=\nu / \kappa=0.25$ in all cases. Here evaluated at midlayer depth are the Rayleigh number $R_{a}=(-\partial \bar{\rho} / \partial \bar{S}) \Delta S g L^{3} / \rho \nu \kappa$ (with $\Delta S$ the entropy contrast across the core), the Taylor number $T_{a}=4 \Omega^{2} L^{4} / \nu^{2}$, the convective Rossby number $R_{o c}=\left(R_{a} / T_{a} P_{r}\right)^{1 / 2}$, and the rms Reynolds number $\tilde{R}_{e}=\tilde{v} L / \nu$, where $\tilde{v}$ is a representative rms convective velocity. A Reynolds number based on the peak velocity at middepth would be about 4 times larger. The eddy viscosity $\nu$ and eddy conductivity $\kappa$ at the middle of the core are quoted in $\mathrm{cm}^{2} \mathrm{~s}^{-1}$.

Further, the extent of penetration of the convection into the surrounding stable radiative envelope varies from equator to pole, yielding a prolate shape to the central region of nearly adiabatic stratification.

The rich time dependence typical of core convection implies that extended simulation runs must be conducted in order to sample the dynamics. Attaining a statistically equilibrated state in the dynamics typically requires evolving the flows for about 1500 days of physical time, or roughly 60 rotational periods at the nominal solar rate. Deductions about the mean flows accompanying the convection, such as differential rotation and meridional circulation, or of the mean heat transport achieved by the convection require averaging the flows over lengthy periods of time, typically greater than 100 days in the late stages of a simulation. We begin by describing some of the general attributes of the convection realized in our simulations.

\subsection{Topology and Scales of Convective Flows}

Core convection clearly involves global-scale overturning motions that extend over much of the unstable domain. This is illustrated in Figure 2, which presents both a three-dimensional rendering of the instantaneous radial velocity near the outer surface of the convective core studied in case $\mathrm{E}$ and an equatorial cut at the same instant through that core domain. Specific regions of upflow and downflow occupy large fractions of the irregular surface (Fig. 2a) and extend in radius over much of that unstable volume (Fig. $2 b$ ). Evidently, there is global connectivity in the flows over well-separated locations, spanning wide ranges in longitude and latitude in both hemispheres. The equatorial cut reveals that the flow patterns involve a prominent azimuthal wavenumber $m=2$ component, although many other wavenumbers are certainly also involved in describing these overturning motions. Little asymmetry is apparent between upflows and downflows: radially inward and outward motions occur on roughly the same spatial scales and possess comparable flow amplitudes (see also Table 2). This appears to be a consequence of the relatively modest radial density contrast (about 2.5) present across the unstable core. We do not here obtain the fast narrow downflows and much broader upflows realized in far more compressible configurations representative
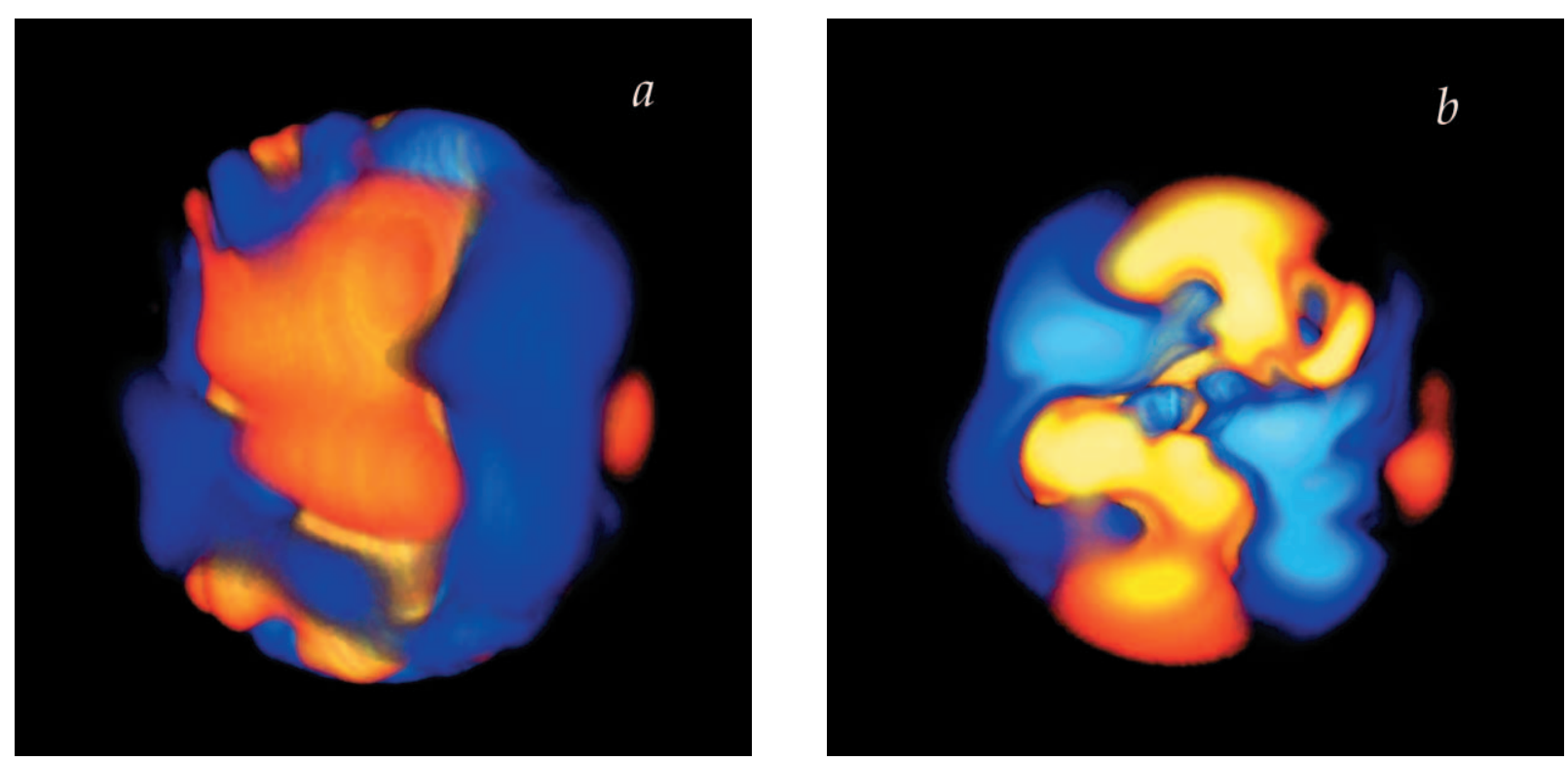

FIG. 2.- Radial velocity at one instant in time for case E, using volume rendering of flow structure $(a)$ near the outer boundary of the prolate convective core, with

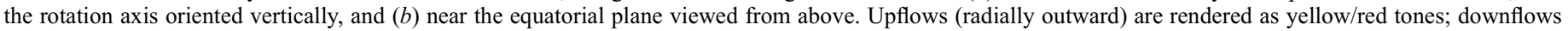
are bluish. 
TABLE 2

Velocities, Energies, Differential Rotation, and Overshooting

\begin{tabular}{|c|c|c|c|c|c|c|}
\hline Parameter & A & B & $\mathrm{C}$ & $\mathrm{D}$ & E & $\mathrm{C} 4$ \\
\hline$\tilde{v}_{r}$ & 36 & 15 & 28 & 14 & 30 & 26 \\
\hline$\tilde{v}_{\theta}$ & 27 & 24 & 26 & 22 & 26 & 21 \\
\hline KE ...................................... & $1.0 \times 10^{8}$ & $3.3 \times 10^{7}$ & $7.6 \times 10^{7}$ & $2.2 \times 10^{7}$ & $7.4 \times 10^{7}$ & $1.2 \times 10^{8}$ \\
\hline 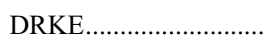 & $5.6 \times 10^{7}$ & $3.5 \times 10^{6}$ & $4.6 \times 10^{7}$ & $5.6 \times 10^{6}$ & $4.3 \times 10^{7}$ & $1.0 \times 10^{8}$ \\
\hline CKE. & $4.2 \times 10^{7}$ & $2.9 \times 10^{7}$ & $2.9 \times 10^{7}$ & $1.6 \times 10^{7}$ & $3.0 \times 10^{7}$ & $1.3 \times 10^{7}$ \\
\hline 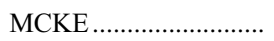 & $2.5 \times 10^{6}$ & $5.4 \times 10^{4}$ & $8.6 \times 10^{5}$ & $2.4 \times 10^{4}$ & $1.1 \times 10^{6}$ & $3.7 \times 10^{5}$ \\
\hline$\Delta \Omega / \Omega_{0} \ldots \ldots \ldots \ldots \ldots \ldots \ldots \ldots$ & $65 \%$ & $26 \%$ & $56 \%$ & $28 \%$ & $62 \%$ & $36 \%$ \\
\hline $\bar{d}_{0} \ldots \ldots \ldots \ldots \ldots \ldots \ldots \ldots$ & $3.41 \times 10^{9}$ & $3.35 \times 10^{9}$ & $2.20 \times 10^{9}$ & $3.25 \times 10^{9}$ & $1.75 \times 10^{9}$ & $2.57 \times 10^{9}$ \\
\hline$r_{c}^{e}$ & $1.93 \times 10^{10}$ & $1.82 \times 10^{10}$ & $1.80 \times 10^{10}$ & $1.89 \times 10^{10}$ & $1.76 \times 10^{10}$ & $1.89 \times 10^{10}$ \\
\hline$r_{c}^{p}$ & $2.16 \times 10^{10}$ & $1.97 \times 10^{10}$ & $2.05 \times 10^{10}$ & $2.03 \times 10^{10}$ & $2.00 \times 10^{10}$ & $2.05 \times 10^{10}$ \\
\hline $\bar{r}_{o}$ & $2.34 \times 10^{10}$ & $2.24 \times 10^{10}$ & $2.19 \times 10^{10}$ & $2.10 \times 10^{10}$ & $2.07 \times 10^{10}$ & $2.28 \times 10^{10}$ \\
\hline
\end{tabular}

Notes.-Components of rms velocity components $\tilde{v}_{r}$ and $\tilde{v}_{\theta}$ at midcore depth $(r=0.10 R)$ are expressed in meters per second. The temporal averages over the full domain of the kinetic energy density (KE), and the energies associated with the differential rotation (DRKE), convection (CKE), and meridional circulation (MCKE), are all expressed in ergs $\mathrm{cm}^{-3}$. The relative angular velocity contrasts $\Delta \Omega / \Omega_{0}$ between $0^{\circ}$ and $60^{\circ}$ latitude are stated. The geometry of the prolate convective core and of the surrounding region of overshooting is measured by the radius of the convectively unstable region at the equator $\left(r_{c}^{e}\right)$ and at the poles $\left(r_{c}^{p}\right)$, the outer boundary of the region of overshooting $\left(\bar{r}_{o}\right)$, and the spherical average of the spatial extent of the overshoot $\left(\bar{d}_{o}\right)$, all in centimeters.

of the solar convection zone (Brummell, Hurlburt, \& Toomre 1998; Brun \& Toomre 2002).

The convectively unstable region that results is mildly prolate in shape (Fig. 2a), having greater spatial extent near the poles than near the equator. This nonspheroidal shape (see $\S 6.1)$ for the extent of the vigorous convection is a striking feature shared by all our simulations. The rumpled appearance of $v_{r}$ in this three-dimensional volume rendering comes from emphasizing the large-scale penetrative structure of such velocity fields. In studying time sequences of such renderings, the interface between the well-mixed interior and the radiative exterior looks much like a throbbing heart, as penetrative motions wax and wane and propagate laterally, launching gravity waves in the process (see $\S 5.2$ ). Analysis of the time evolution of equatorial cuts (Fig. $2 b$ ) reveals that some flows dive through the central regions of the core to emerge on the other side, with such patterns exhibiting distinctive retrograde motion.

\subsection{Velocity and Thermal Patterns}

We present in Figure 3 global mappings for cases B and C of both the radial velocities $v_{r}$ and temperature fluctuations $T$ (relative to the average of temperature on a spherical surface at a given radius). These Mollweide projections for the fields are shown both well within the convective interior (at $r=0.10 R$; Fig. 3, lower panels) and at the transition into the stable exterior $(r=0.16 R$; Fig. 3, upper panels). These mappings reveal that the radial velocity patterns in any given snapshot have a noticeable alignment with the rotation axis. (Such projections exhibit meridian lines as increasingly curved arcs away from the central meridian, which appears as a vertical line; here the lines of constant latitude are indeed parallel.) The more laminar case B (Figs. $3 a-3 d$, left) possesses structures that are largely symmetric about the equator, whereas the lower diffusivities $(\nu$ and $\kappa)$ in case C (Figs. 3e-3h, right) lead to flows with broken symmetries in the two hemispheres as more intricate flows are realized. In both cases the flow structures at high latitudes are more isotropic and of smaller scale, and somewhat faster flows are realized for case C (see also Table 2).

Radial motions in these simulations are intimately linked to fluctuations in temperature. These buoyantly driven flows should typically involve positive $T$ in regions of ascending motion within the unstable core and negative $T$ in descending flows there. Such a positive correlation between $v_{r}$ and $T$ is largely realized in the lower panels in Figure 3 for both cases showing the $r=0.10 R$ depth where the convection is vigorous. The less complex flows in case B exhibit such correlation readily, whereas in case $\mathrm{C}$ there are also sites where the sense of the correlation is reversed. The nature of the correlations changes as motions penetrate into the radiative surroundings. The buoyancy braking that is expected within this region of stable stratification, as at the $r=0.16 R$ depth (Fig. 3, upper panels), arises from the radial velocities and temperature fluctuations becoming anticorrelated. The sense of $T$ in both regions is partly controlled by the source term in equation (4) that involves the product of $v_{r}$ with the radial mean entropy gradient. When the latter gradient changes sign in going from an unstable stratification (superadiabatic in the convective core) to stable (subadiabatic) stratification as flows extend upward into the radiative envelope, so does the sense of $T$. This correspondence between radial velocities and temperature fluctuations is true only in a general sense: on fine scales, secondary instabilities can drive motions that locally violate these principles. Similarly, the nonlinear advection of temperature fluctuations by motions that twist and turn can lead to unusual combinations of $v_{r}$ and $T$ at some sites. The anticorrelation of $v_{r}$ and $T$ in the region of penetration is clearest in case $B$ involving the simpler flow fields. Because the thermal diffusivity is larger than the viscosity in these simulations (i.e., the Prandtl number $P_{r}$ is less than unity, namely, 0.25), thermal structures have a somewhat larger physical scale than do associated features in the radial velocity.

The temperature fluctuations at both depths in Figure 3 have amplitudes of only a few kelvins (compared with the mean temperatures of millions of kelvins found at such depths), which, combined with radial velocities of order $50 \mathrm{~m} \mathrm{~s}^{-1}$ in the bulk of the convective interior and the high heat capacity of the gas, are sufficient to carry outward a good fraction of the emerging flux (see $\S 3.3$ ). The radial velocities have plummeted to much smaller values just beyond the edge of the convection zone where the motions are beginning to penetrate upward into the radiative zone.

The convective fields in these simulations can be assessed using probability distribution functions (PDFs). Figure 4 shows PDFs for the radial velocity and temperature fluctuations on a 

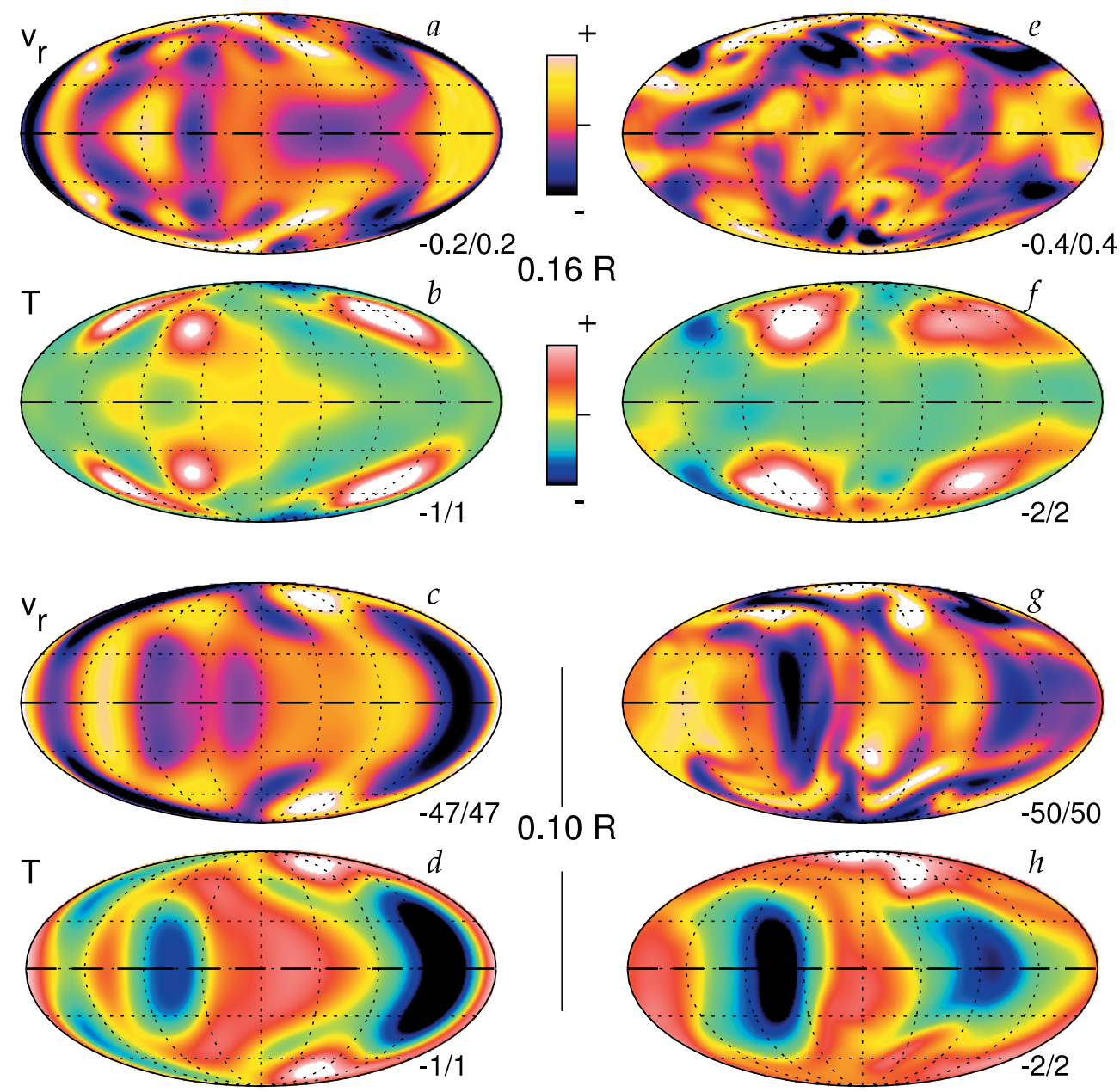

FIG. 3. - Global mappings of radial velocities $\left(v_{r}\right)$ and associated temperature fluctuations $(T)$ at one instant in time for the laminar case B (left) and the more

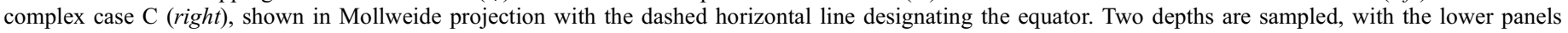

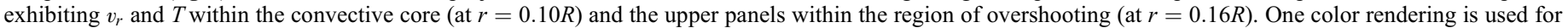
$v_{r}$ and another for $T$, with the maximum and minimum amplitudes for each field (in meters per second and kelvins) indicated next to each panel.

spherical surface within the convective core $(r=0.10 R)$ and the region of overshooting $(r=0.16 R)$ of case $\mathrm{C}$, exhibiting both instantaneous PDFs and time-averaged ones. The distributions of radial velocities are largely symmetric Gaussian functions at both depths. The detailed features in the instantaneous PDFs change as the flows evolve. The instantaneous temperature field distributions (Figs. $4 d$ and $4 h$ ) possess spikes, indicating the presence of large-scale coherent temperature structure; at other instants, the spikes may be replaced by broader plateaus. The time-averaged PDFs for $T$ are nonsymmetric, with broad tails extending to positive values in the region of overshooting and to negative ones in the core. These tails serve to compensate for the off-center most probable values that are positive in the core and negative in the overshooting region. Such complexities in the PDFs are realized in all of our simulations and thus are at some variance with simpler relations assumed in mixing-length treatments of convection.

\subsection{Energy Transport}

The release of energy by CNO cycle nuclear burning in the core leads to a progressively increasing local luminosity with radius within the inner parts of our domain. The energy produced in such a distributed fashion gets carried outward primarily by radiative diffusion and mechanical transport of heat (or enthalpy) by the convection. To assess the role convection plays in the overall energy balance, we recall that the rate at which convection transports energy in the radial direction is given by the enthalpy flux

$$
F_{e}(r)=\bar{\rho} c_{p} \overline{v_{r} T}
$$

involving a product of the radial velocity with fluctuations in the heat content of the fluid, proportional to $T$, and averaged over a spherical surface and in time (indicated by the overbar). We may compare $F_{e}$ with the flux of energy carried by radiation,

$$
F_{r}(r)=-\kappa_{r} \bar{\rho} c_{p}\left(\frac{d \bar{T}}{d r}+\frac{\overline{d T}}{d r}\right),
$$

with the latter term arising from the mean of radial gradients of temperature fluctuations, which are here negligible. The enthalpy and radiative fluxes largely suffice to carry outward the energy produced by nuclear reactions within the core, yielding a total flux

$$
F_{t}=\frac{L(r)}{4 \pi r^{2}}
$$

where $L$ is the luminosity of the star, which increases with radius until the surface luminosity $L_{*}$ is attained. (The kinetic 

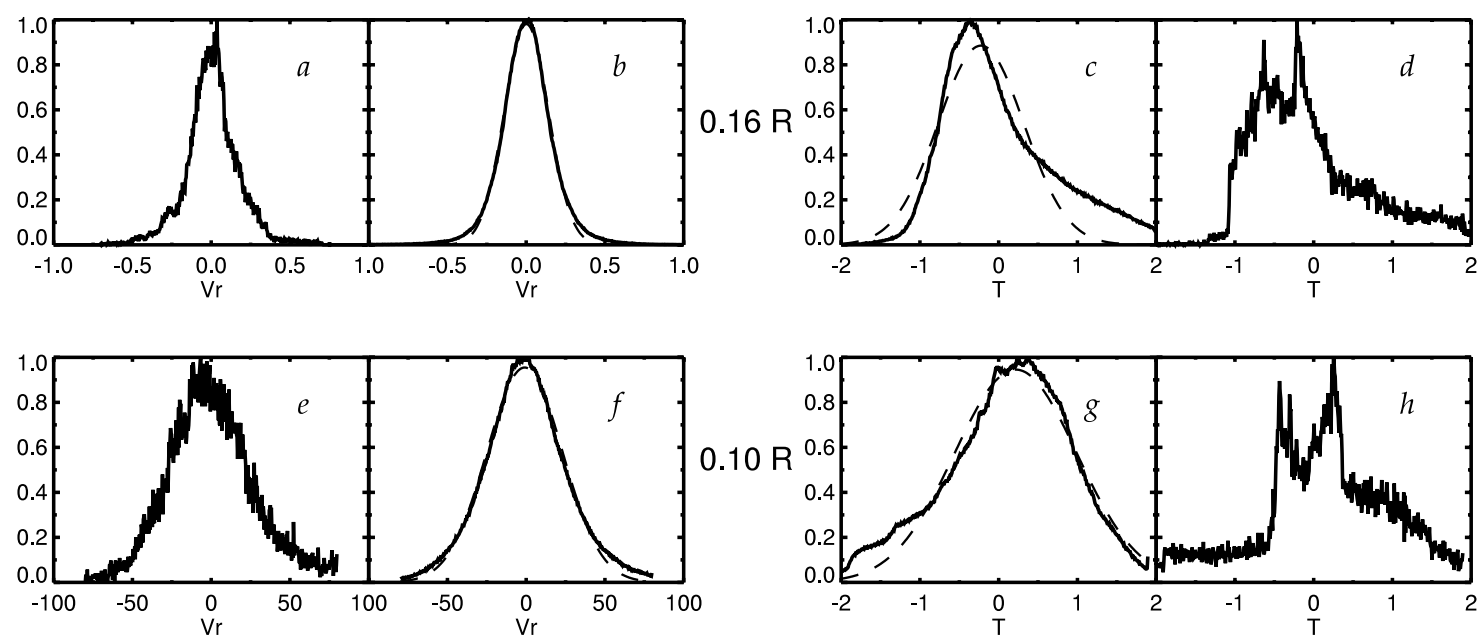

FIG. 4.-Probability distribution functions in case $\mathrm{C}$ for radial velocities $(l e f t)$ and temperature fluctuations (right) within the convective core $(e-h$; at $r=0.10 R)$ and the region of overshooting $(a-d$; at $r=0.16 R)$. $(a, d, e, h)$ Instantaneous PDFs for the two fields; $(b, c, f, g)$ temporal averages over about 180 days together with

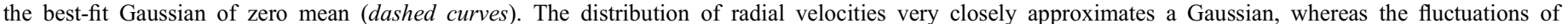
temperature are slightly asymmetric about their most probable value.

energy flux, the viscous flux, and the flux carried by unresolved subgrid motions are all small compared with $F_{e}$ and $F_{r}$.)

Figure 5 displays the variation with radius of $F_{r}$ and $F_{e}$, expressed as luminosities, for case $\mathrm{C}$. The enthalpy flux is maximized near the middle of the convective core (at $r=0.08 R$ ), serving to carry about $57 \%$ of the local stellar luminosity $L$ there, with the remainder transported by radiation. Since the core convection establishes a nearly adiabatic stratification (with $\nabla-\nabla_{\text {ad }} \sim 10^{-7}$ ), the associated temperature gradient that arises serves to specify a radiative flux $F_{r}$ that increases steadily with radius. Such a growing $F_{r}$ forces $F_{e}$ to decrease in the upper half of the unstable core. Beyond the convectively unstable region, at $\bar{r}_{c} \sim 0.14 R$ (see $\S 6.2$ ), the enthalpy flux becomes negative, resulting from the anticorrelation of radial velocity and temperature fluctuations as the motions are braked. The radiative flux there must compensate for that inward directed enthalpy flux. Our simulations

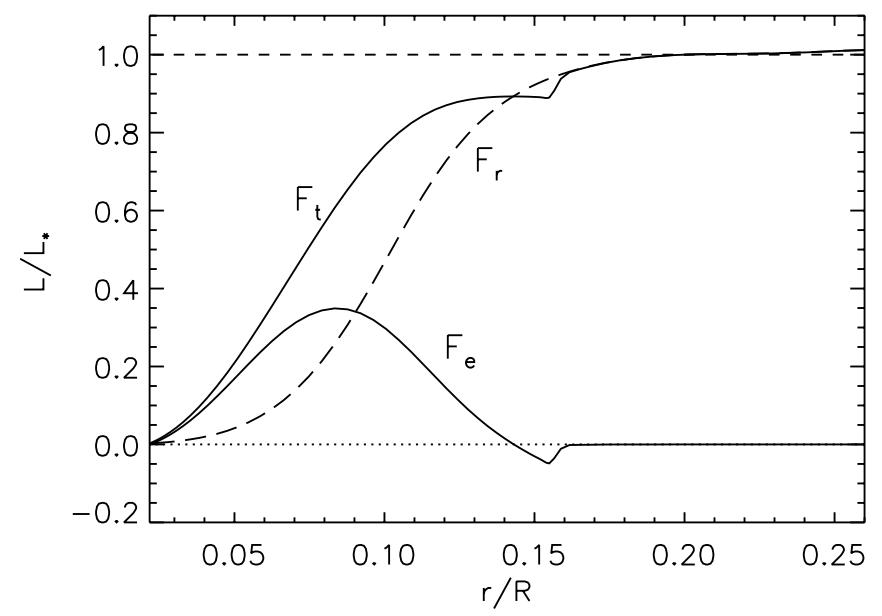

FIG. 5.-Time-averaged radial transport of energy within case $\mathrm{C}$ as a function of proportional radius, formed over an interval of about 180 days. Shown are the enthalpy flux $F_{e}$ and the radiative flux $F_{r}$, together with their sum, the total flux $F_{t}$; all quantities have been expressed as luminosities. The convective core extends here to about $r=0.14 R$, with the positive $F_{e}$ there serving to carry as much as $57 \%$ of the total flux. The farther region of overshooting involves a small negative (inward directed) enthalpy flux. have not been evolved for a sufficient amount of time to allow this compensation to occur fully, since the relevant thermal relaxation time is long compared with other timescales in the problem. Thus, the total luminosity in Figure 5 displays a small dip at the interface between the convective and radiative zones, which in real stars (or a fully evolved simulation) would be absent.

The correlations of radial velocity and fluctuating temperature that yield an outward-directed enthalpy flux are achieved in a complicated manner within the bulk of the convective interior. In mapping these variables at one instant in time, it is evident that a number of sites yield an unfavorable correlation, whereas in the main the requisite positive correlation is realized.

The nearly adiabatic stratification achieved by the convection in the bulk of the core is much as in one-dimensional stellar models employing standard mixing-length treatments. However, our self-consistent three-dimensional analysis of convective dynamics and its transports also provides estimates of the stratification in the region of overshooting, which are far more difficult to assess using mixing-length approaches.

\section{RESULTING DIFFERENTIAL ROTATION}

Convection occurring within deep spherical domains should be strongly influenced by the rotation of the star when the convective overturning time is of the order of or greater than the rotation period. This implies a convective Rossby number $R_{o c}$ (see Table 1) that is of order unity or smaller. The global-scale convection in our models has an overturning period of about 1 month, comparable to the rotation period of a star rotating at the solar rate. Thus, we expect the convection influenced by Coriolis forces to possess correlations between the components of its velocity field. The resulting Reynolds stresses can serve to continuously redistribute angular momentum, leading to angular velocities within the core that are far from uniform rotation. Further, the connected topology of the convective core appears to play an important role in the prominent differential rotation profiles that are realized in our simulations.

\subsection{Central Column of Slow or Rapid Rotation}

We find that strong angular velocity contrasts are established within the convective cores in all our simulations. For model 
stars that rotate at least as rapidly as the Sun, these involve roughly cylindrical central columns of decidedly slow rotation (retrograde relative to the reference frame), accompanied by equatorial regions of somewhat enhanced rotational velocity (prograde). As shown in Figure 6 (left) for the three cases B, C, and $\mathrm{C} 4$, the time-averaged mean longitudinal velocity $\hat{v}_{\phi}$ exhibits some variations along that central column, with those fluctuations becoming more pronounced as the complexity of the convection increases in going from case B to C (see Fig. 3). There is likewise greater asymmetry in $\hat{v}_{\phi}$ between the two hemispheres (delineated by the equator) in these cases. Such symmetry breaking may be anticipated as the convection becomes increasingly nonlinear. The convection itself is not symmetric about the equator, and thus mean flows associated with it, including the differential rotation, are likely to exhibit differences in the two hemispheres. The columns of slow rotation extend slightly into the radiative envelopes at high latitudes, in keeping with the prolate shape of the convective core. The presence of the central slow cylinder of rotation does not appear to result from the small inner sphere omitted from our computational domain, for we have found similar columns of slowness for several different sizes of central spheres. Figure 6 (right) shows the variation of angular velocity $\hat{\Omega}$ with radius in three latitudinal cuts, providing another assessment of the slowness of the central column and of the speeding up of the equatorial region.

These central columns of slow rotation are largely unexpected and constitute a striking finding of these simulations. A general result from previous studies of convection in deep shells under strong rotational constraints (with $R_{o c}$ small) is that equatorial regions of fast rotation are obtained (e.g., Gilman 1979; Miesch et al. 2000; Brun \& Toomre 2002). The conservation of angular momentum requires that other regions be slowed down, and this often appears as higher latitude regions of slowness. The analog in our spherical domain may be the central column of particularly retrograde $\hat{v}_{\phi}$, for this could compensate for the equatorial speeding up (prograde $\hat{v}_{\phi}$ ) of regions with a large moment arm. The continuous redistribution of angular momentum by the convection, involving various effects of Reynolds stresses, meridional circulations, and viscous stresses, is examined in $\S 8$ as we seek to understand the balances needed to account for such central columns of slowness.

We have also considered a more slowly rotating case $\mathrm{F}$, with a rotation rate of $\Omega_{0} / 10$, to examine whether the central column of slowness is still achieved under a weaker rotational influence (with $R_{o c}$ large). The prior studies of convection in shells (Gilman 1978) indicated that under such conditions the equatorial regions may exhibit relatively slow rotation due to the modified transport of angular momentum. If this property of shell convection were to carry over to full spherical domains, then it might follow that the overall conservation of angular momentum could yield central regions of fast rotation to compensate for the equatorial slowness. Figure 7 shows that for case $\mathrm{F}$ this is indeed realized. The central region of relatively fast rotation is decidedly less columnar than those of slow rotation in Figure 6, probably because the Taylor-Proudman constraint is weakened. We thus suspect that central columns of slowness may be ubiquitous for stars that rotate sufficiently rapidly that $R_{o c}$ is less than unity. In the remainder of this paper, we concentrate on our simulations that satisfy this criterion (all but case F), since real A-type stars are generally quite rapidly rotating.
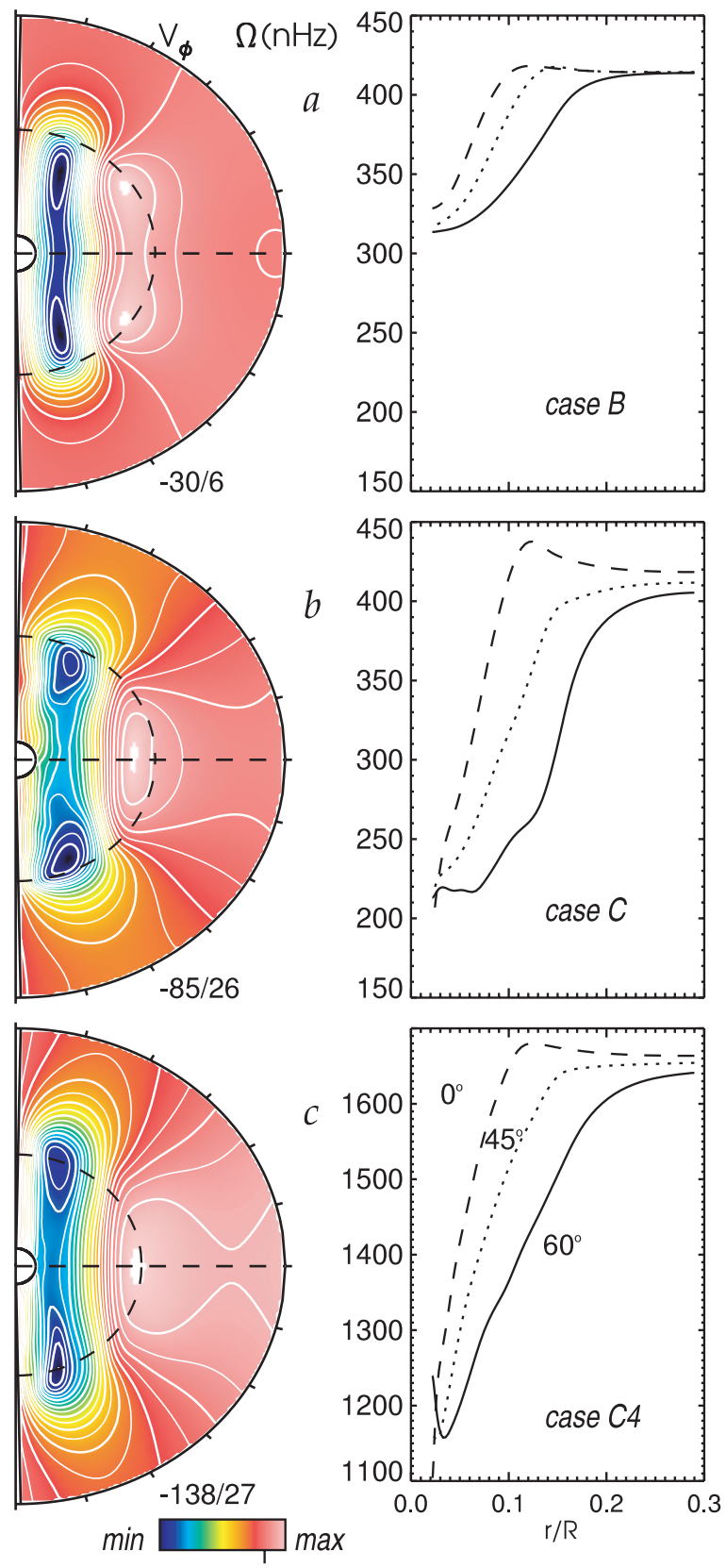

FIG. 6.- Strong differential rotation established by the convection in the three cases B, C, and C4. Shown are the azimuthal velocity $\hat{v}_{\phi}$ as contour plots in radius and latitude (left; with dashed line denoting equator) and the angular velocity $\hat{\Omega}$ with proportional radius for three latitudinal cuts (right); both $\hat{v}_{\phi}$ and $\hat{\Omega}$ are averaged in time (all over intervals of roughly 110 days) and longitude. Maxima and minima of $\hat{v}_{\phi}$ (in meters per second) are indicated. Cases B (top) and C (middle) are rotating at the solar rate $(414 \mathrm{nHz})$, and case $\mathrm{C} 4$ (bottom) rotates 4 times faster. Greater angular velocity contrasts are achieved in the more complex flows of case $\mathrm{C}(b)$ and case $\mathrm{C} 4(c)$ than in the laminar case B $(a)$, in all cases involving a central column of slowness.

\subsection{Contrasts in Angular Velocity}

We now turn to the time-averaged angular velocity $\hat{\Omega}$ profiles associated with the differential rotation in our convective cores with $R_{o c}$ small, some examples of which are shown as radial cuts at constant latitudes in the right-hand panels of Figure 6 that accompany the views of $\hat{v}_{\phi}$. The $\hat{\Omega}$ profiles in Figure 6 emphasize that most of the radiative envelope rotates at the 

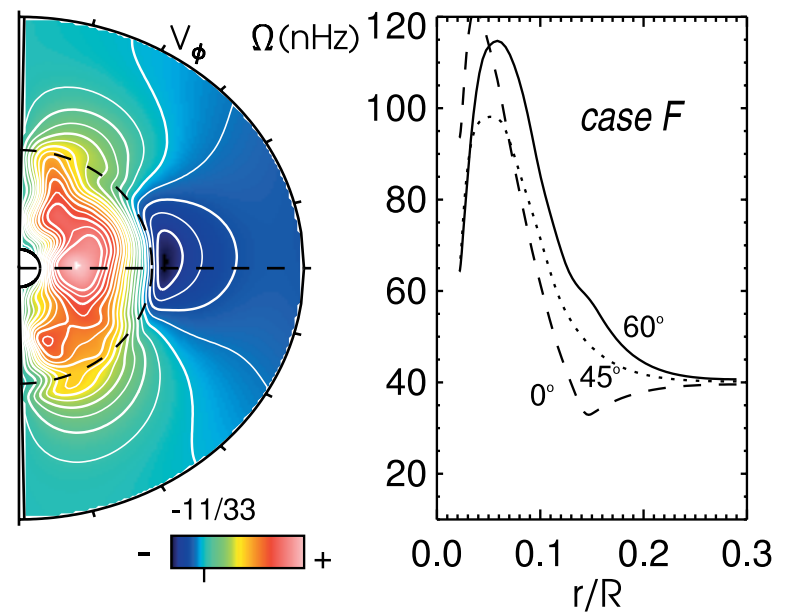

FIG. 7.-Fast column of central rotation realized in case F rotating at 1/10 the solar rate. Shown is the azimuthal velocity $\hat{v}_{\phi}$ as a contour plot in radius and latitude (left; with dashed line denoting equator) and the angular velocity $\hat{\Omega}$ with proportional radius for three latitudinal cuts (right); both $\hat{v}_{\phi}$ and $\hat{\Omega}$ are averaged in time (over 180 days) and longitude. Maxima and minima of $\hat{v}_{\phi}$, in meters per second, are indicated.

nominal rate of the reference frame. The variations in $\hat{\Omega}$ are largely confined to the convective core, although within the overshoot region there are still some weak gradients in $\hat{\Omega}$ evident. In the simulations of semiturbulent convection rotating at the solar rate (cases A, C, and E, with identical viscosities but increasing "stiffness" in the stratification of the radiative exterior), we obtain angular velocity contrasts $\Delta \hat{\Omega} / \Omega_{0}$ (from the equator to $60^{\circ}$ ) of order $60 \%$ (see also Table 2). These contrasts appear to be insensitive to the stratification of the radiative zone considered here; likewise, the character of the differential rotation throughout the convective core is similar in these three cases. In more laminar simulations at the same rotation rate (cases $\mathrm{B}$ and $\mathrm{D}$, involving greater viscosities than their companion cases $\mathrm{C}$ and $\mathrm{E}$ ), although the angular velocity contrast is lessened to about $25 \%$, a central cylinder of slow rotation is still evident. At a fourfold higher rotation rate, considered as case $\mathrm{C} 4, \Delta \Omega$ increases by about a factor of 2 , yielding a $\Delta \Omega / \Omega_{0}$ that is reduced to about $33 \%$; a central column of slowness is retained. As we increased the rotation rate in going to case $\mathrm{C} 4$ from case $\mathrm{C}$, we sought to maintain roughly the same degree of supercriticality in the convection, which required decreasing the effective viscosities to compensate for the stabilizing effects of rotation (e.g., Chandrasekhar 1961). Without such decreases, the angular velocity contrast was lessened to about $22 \%$ in the resulting more laminar flow.

Another assessment of the resulting differential rotation in these simulations is provided by examining the kinetic energies associated variously with the convection (CKE), meridional circulation (MCKE), and differential rotation (DRKE), together with their sum (KE), all relative to the rotating frame. As detailed in Table 2, the CKE is reasonably invariant across all our simulations rotating at the solar rate. In going from the more laminar flows of cases B and D to the semiturbulent flows in cases $\mathrm{C}$ and $\mathrm{E}$, the stronger differential rotation that results is reflected in the greater DRKE and thus also in the total kinetic energy. The MCKE is likewise increased but remains a small fraction of KE. Thus, the more complex flows more effectively transfer energy - from the vast reservoirs of energy associated with the rotation of the frame and with the internal energy of the star-into KE, which nonetheless remains a small fraction of the total energy in these simulations.

From our limited sampling of rotation rates, we cannot reliably estimate how $\Delta \Omega / \Omega$ is likely to vary with $\Omega$ at the much higher rates typical of many A-type stars. However, we suspect that the strong differential rotation obtained in our simulations is likely to be realized even for core convection within more rapidly rotating stars with much stronger rotational constraints.

\section{EVOLVING CONVECTION AND WAVE EXCITATION}

The inherently time-dependent convection involves substantial pattern evolution and propagation within the bulk of the convective core and the excitation of gravity waves in the surrounding radiative envelope. Some large features in the convection appear, persist, and are sheared and advected by the mean flows that they drive. The radiative zone contains the rippling signature of unremitting gravity waves excited by the convection below, accompanied by weak circulations. We now consider such time variability.

\subsection{Propagation and Shearing of Convective Structures}

The flows in these simulations evolve in a complicated manner. This may be seen in Figure 8, which examines the radial velocity patterns within the convective core (at $r=0.10 R$ ) in case $\mathrm{C}$ at three instants each separated by 7 days in time. Large-scale features are recognizable in all three images but show evolution and propagation. Some orderly downflow structures within the convection persist for long periods of time. Yet they also clearly evolve over relatively short time intervals: major downflow lanes present in Figure $8 a$, for instance, are recognizable in Figure $8 c$ but have changed in shape and intensity. They do not evolve in isolation: patterns merge, are sheared, and cleave into smaller patterns as they interact with surrounding flows. Such features are not simply advected by the mean motion of the region in which they are embedded but can additionally propagate relative to the differential rotation that they establish. The rate and sense of pattern propagation is most readily seen by turning to timelongitude mappings, such as in sampling the radial velocities at $65^{\circ}$ north (Fig. 8d) and at the equator (Fig. 8e) over nearly 200 days. In these mappings, persistent strong downflow lanes are revealed as dark bands tilted to the right in Figure $8 e$ and sheared to the left in Figure $8 d$. Such tilts reveal that patterns at this radius and near the equator propagate in a prograde sense, taking about 300 days to complete one revolution, and at the higher latitude their propagation is retrograde with a period of about 60 days. These mappings also emphasize that the convection possesses larger scales at the equator than at high latitudes, with a distinctive change in the sense of pattern propagation relative to the frame at midlatitudes.

\subsection{Internal Gravity Wave Responses}

The vigorous core convection extends upward into the stable stratification that bounds it, with such pummeling serving to excite internal gravity waves within the radiative envelope. Such wave responses are shown vividly in the upper panels of Figure 9, which displays the radial velocities at multiple depths in cases E and C4. These can appear either as localized ripples that are one signature of the waves (Fig. 9a), arising either from distinctive events or from the interference of many waves, or as striking large-scale global resonances (Fig. 9d) that persist (once established) for as many rotation 

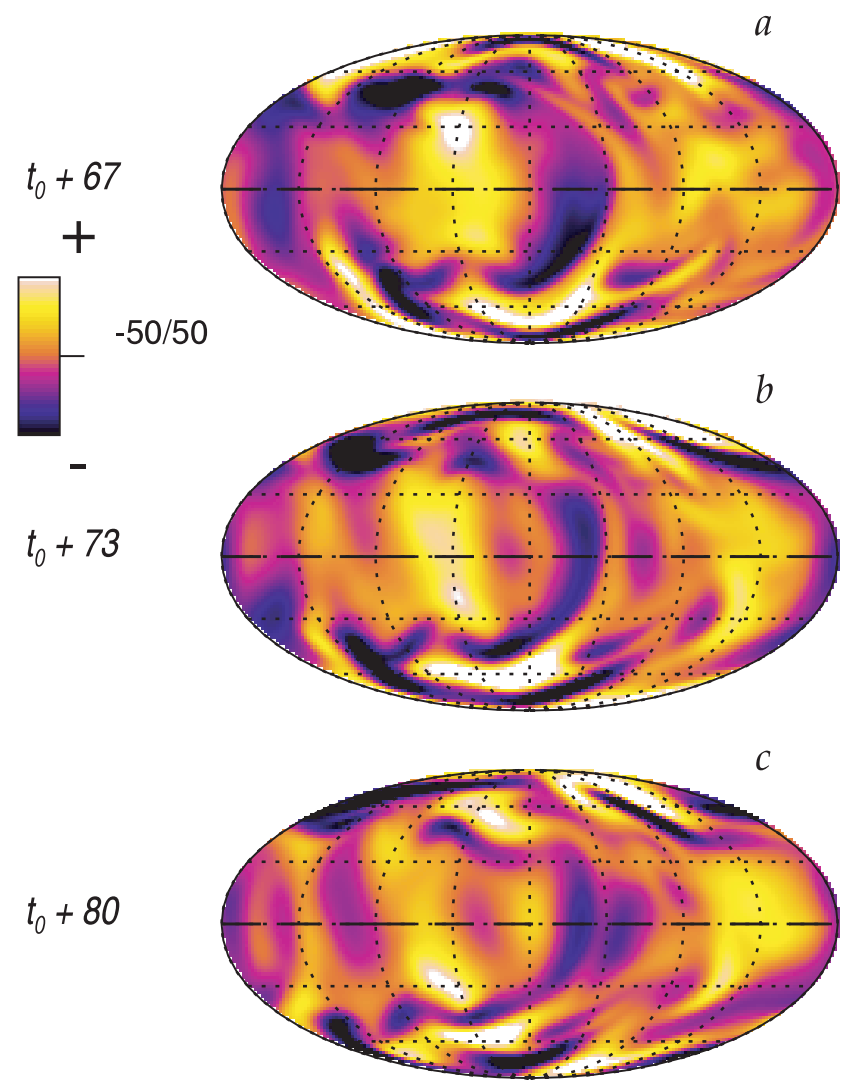

$d$

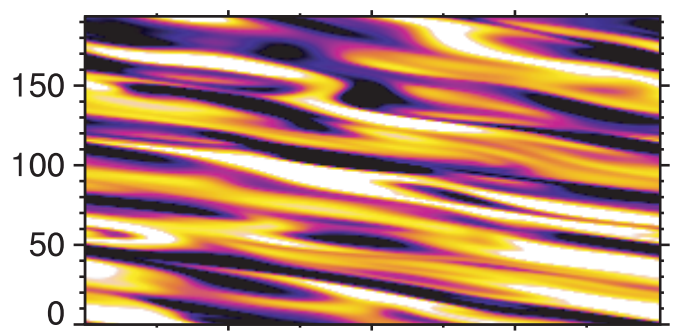

e

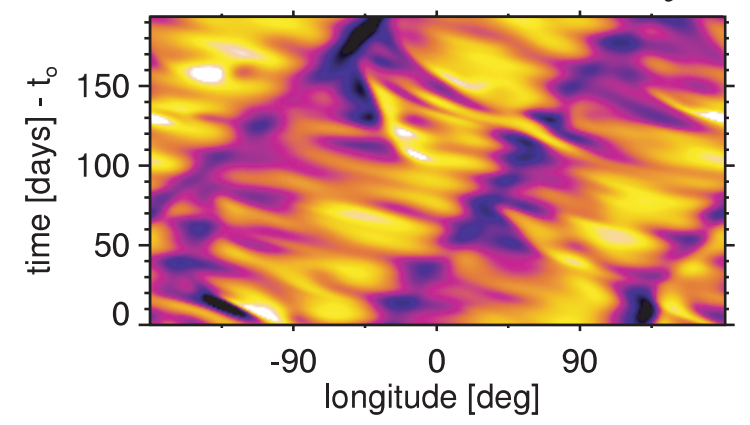

FIG. 8.-Evolution and propagation of the convective flows sampled for case C. $(a-c)$ Global mappings of the radial velocity $v_{r}$ within the convective core (at $r=0.10 R$ ) for three successive times each separated by 7 days, exhibiting shearing and cleaving of the convective structures. The propagation of those convective patterns is most evident in the time-longitude maps of $v_{r}$ extracted at $(d)$ a latitude of $65^{\circ}$ and $(e)$ the equator. The same color rendering is used in all panels.

periods as we continued the simulations. The latter response may be more easily realized in our simulations, with their artificially imposed outer boundary conditions that reflect gravity waves, than in real stars, where the radiative envelope is extensive and probably has no simple reflecting surfaces.
However, they may also mimic gravity waves of moderate radial order $n$ (such as $n=8$ or 10) in a real star, although the stable stratifications in such stars are much stiffer than the ones we consider here.

The generation of gravity waves by the upward-directed plumes is fully expected. Waves with frequencies less than or equal to the Brunt-Väisälä frequency $N$ (proportional to the square root of the local entropy gradient $d \bar{S} / d r$ of the stable stratification) are able to propagate in the radiative outer envelope and may be excited in abundance by the time-varying convection, as in case $\mathrm{E}$ at $r=0.26 R$ (Fig. 9a). This also implies that the spectrum will shift as the stiffness of the surrounding envelope is varied. More surprising is the large-scale global resonance of an internal gravity mode (of spherical harmonic degree $\ell=3$ and azimuthal order $m=2$ ) that is readily achieved within the more rapidly rotating case $\mathrm{C} 4$ and likewise within some of its more laminar relatives. This may come about because the rotating convection is likely to possess a range of inertial oscillations with frequencies less than $2 \Omega_{0}$ (Greenspan 1969), which accompany the broad band of lower frequencies present in the time-dependent convection. When there is a suitable overlap between the temporal spectrum of the rotating convection and the admissible internal gravity wave frequencies that depend on $\ell$ and radial order $n$ (e.g., Unno et al. 1979; Gough 1993; Dintrans, Rieutord, \& Valdettaro 1999), a prominent wave response may result. In our models $N$ varies somewhat with radius; for case $\mathrm{C} 4$ its representative value is $1.1 \times 10^{-5} \mathrm{~Hz}$, whereas $\Omega_{0}$ is $1.04 \times 10^{-5} \mathrm{~Hz}$. It appears that a global gravity wave (with low $\ell$ ) has been selected that propagates retrograde relative to the frame (with a period of about 40 days for a complete revolution), within a region that exhibits almost no differential rotation. In addition to the global mode, other gravity waves appear as ripples near the equator in Figure $9 d$.

\section{CONVECTIVE PENETRATION AND RADIATIVE ENVELOPE}

Convective motions do not stop abruptly at the interface between the convective core and the stable radiative envelope. Rather, outward-moving parcels of fluid possess momentum and therefore must travel some distance into the stable radiative zone before they come to a halt. There are two effects. First, if convective motions are sufficiently vigorous and possess a large enough filling factor, they can create a nearly adiabatic stratification within a portion of the overlying radiative zone that in a one-dimensional stellar structure model would be stably stratified. This may be termed a region of penetration, as discussed in Zahn (1991), Hurlburt et al. (1994), and Brummell, Clune, \& Toomre (2002). Second, there will be a farther region of stable stratification into which convective motions extend but whose entropy gradient they fail to modify significantly. Within such a region of overshooting, outward-moving parcels offluid are braked until they come to a halt. Throughout the domain of penetrative convection and the extended region of overshooting, the fluid motions should be effective in mixing the chemical composition.

\subsection{Shape of Convective Core}

In the one-dimensional stellar models used for our initial conditions, the region of unstable stratification is of course spherical. In the evolved simulations, penetrative convective motions have yielded a nearly adiabatically stratified core region (weakly superadiabatic) that is now prolate in shape 

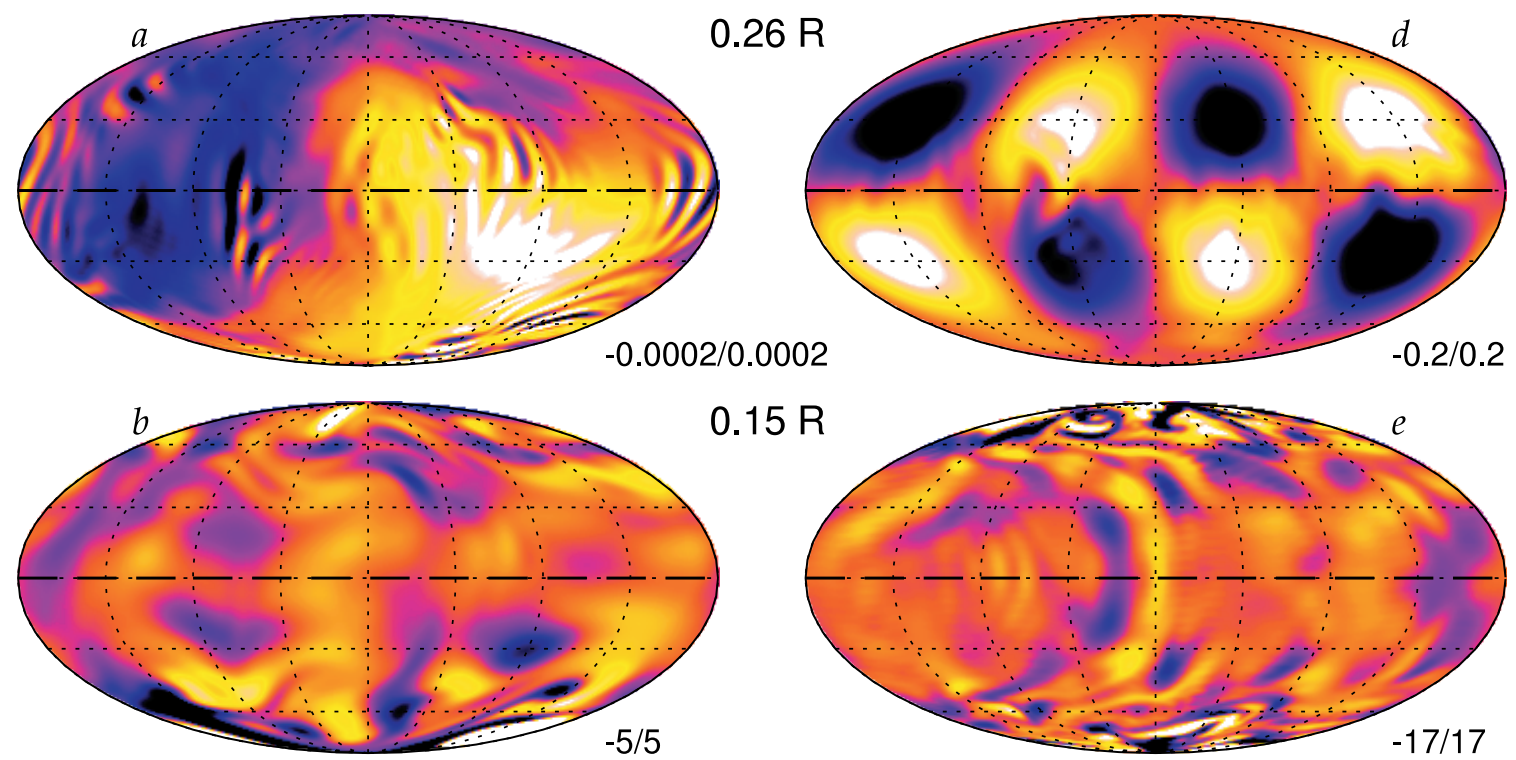

$0.15 \mathrm{R}$
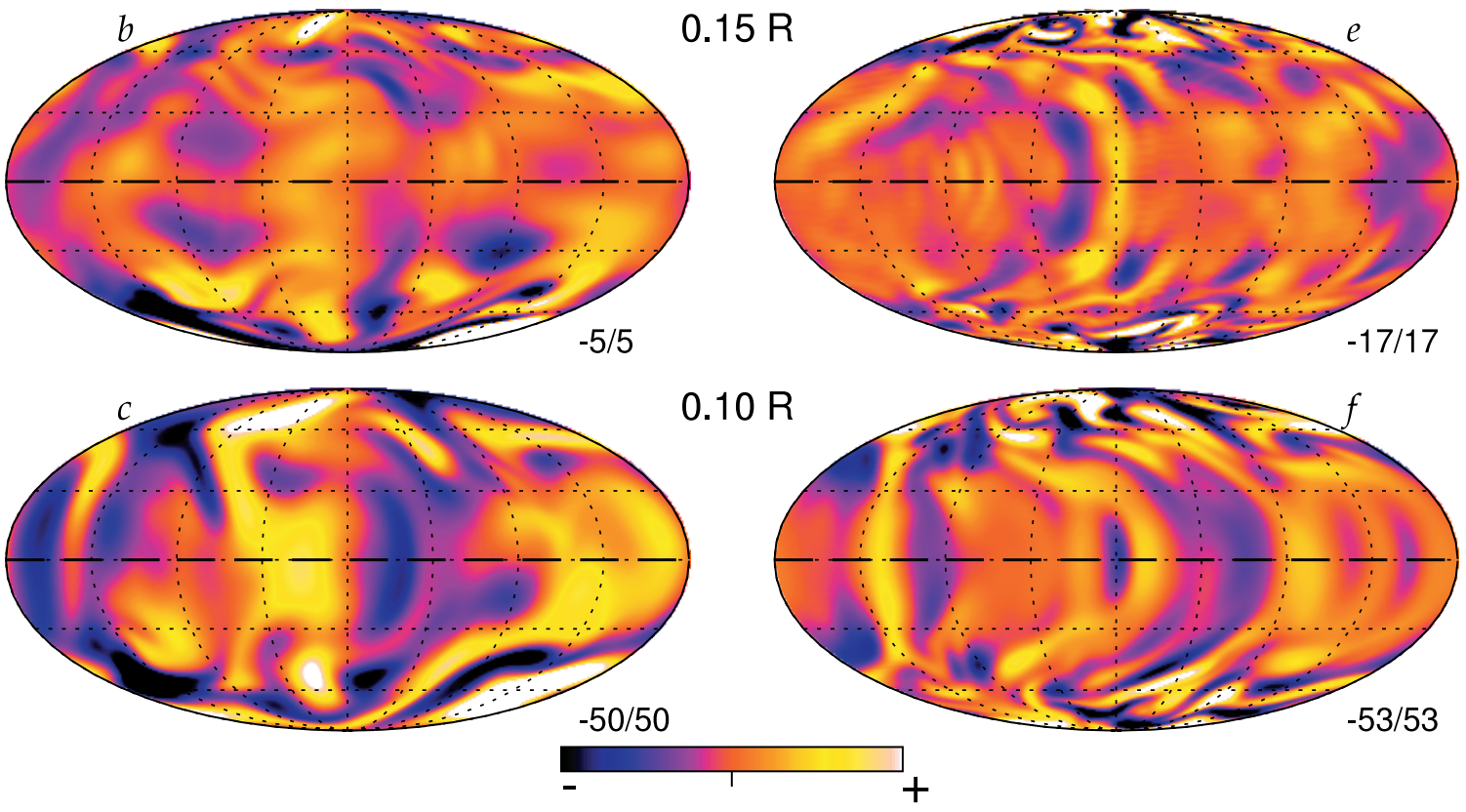

FIG. 9.-Excitation of gravity waves in the radiative envelope for cases E (left) and C4 (right), shown in global mappings of radial velocity $v_{r}$ at one instant in time (top; sampling $r=0.26 R$ ). The associated convective flows are also shown within the overshoot region (middle; $r=0.15 R$ ) and in the convective core (bottom; $r=0.10 R$ ). For case $\mathrm{E}$, gravity waves of small amplitude (scaling indicated) are visible in $(a)$ as weak ripples in the largely quiescent radiative zone. For case $\mathrm{C} 4$, the waves appear in $(d)$ as a prominent global resonance of greater amplitude.

and aligned with the rotation axis. The radius $r_{c}$ of this core region thus varies with latitude, as sketched in Figure 10. The simulations further reveal that the overshooting motions extend outward to a radius $r_{o}$ that is largely independent of latitude and thus roughly spherical in shape, as also sketched.

Such geometries for the penetrative core and the further region of overshooting are shown in Figure 11 for case C. These regions are delineated by turning to the time- and longitude-averaged enthalpy flux, given by

$$
\hat{F}_{e}(r, \theta)=\bar{\rho} c_{p}\left\langle v_{r} T\right\rangle_{t, \phi},
$$

where the angle brackets and their subscripts indicate the averaging. Within the convective core that includes the region of penetration, the enthalpy flux is typically positive, because of the positive correlations of radial velocity and temperature fluctuations that are largely realized there (see $\S 3.2$ ). As the motions extend outward into the subadiabatic stratification of the region of overshoot, the enthalpy flux becomes negative, since the temperature fluctuations typically flip their sign almost immediately, becoming in the main anticorrelated with the radial velocity. Thus, the inner boundary of the region of overshoot is usefully also revealed by the radius at which $\hat{F}_{e}$ changes sign.

The outer boundary $r_{o}$ of that overshooting region is taken to be the radius at which the enthalpy flux has attained an amplitude of $10 \%$ of its most negative value. We introduce this definition because small negative values of the enthalpy flux persist wherever any radial motions anticorrelated with temperature fluctuations exist, even if these radial motions are very weak. Our intent is to identify regions where overshooting motions are reasonably vigorous, recognizing that such a constraint on the amplitude of the enthalpy flux is somewhat arbitrary. For case $\mathrm{C}, r_{o}$ has a value of about $0.158 R$, independent of latitude, whereas $r_{c}$ ranges from about $0.131 R$ at the equator to $0.148 R$ at the poles (Fig. 11). The values for other cases are presented in Table 2. Since in all our cases the inner boundary $r_{c}$ of the overshoot region is a function of latitude and the outer boundary $r_{o}$ is largely not, the extent of the overshooting $d_{o}$ varies with latitude, possessing the greatest spatial extent near the equator. Figure 11 also shows that the enthalpy flux $\hat{F}_{e}$ has prominent variations with latitude and radius within the convective core, with a greater flux at mid- and high latitudes than at the equator. As the convection evolves, the time-averaged $\hat{F}_{e}$ as determined over different epochs shows some changes in its structure and its symmetries with respect to the equator.

The prolate geometry of the convective core may result from the effects of rotation yielding convective flows that typically involve greater radial velocities at high latitudes than at the equator. Figure 12 shows the rms radial velocity in case $\mathrm{C}$ within both the convective core $(r=0.10 R)$ and the region 


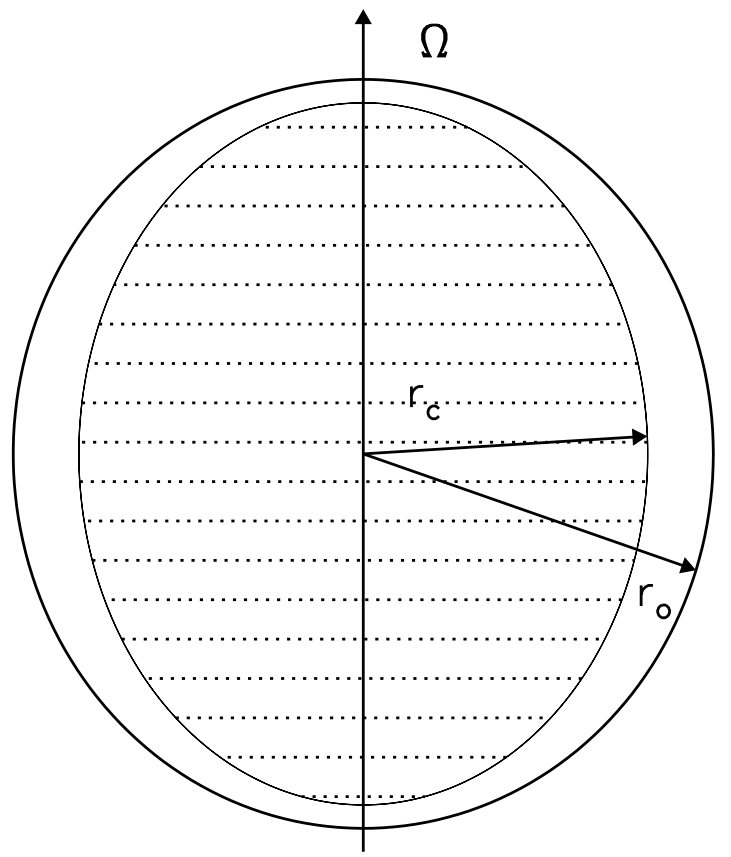

FIG. 10.-Schematic of the typical geometry of the prolate convective core of radius $r_{c}(\theta)$ and of the farther region of overshooting, which extends to radius $r_{o}$ largely independent of latitude $\theta$. The nearly adiabatic convective interior has greatest spatial extent along the axis of rotation (here vertical).

of overshooting $(r=0.15 R)$. It is evident that radial velocities are generally lowest near the equator and highest near the poles. Outward-directed motions at low latitudes are turned by the Coriolis forces, thus decreasing their radial velocity component; radial motions that are closely aligned with the rotation axis, such as at high latitudes, are deflected less and thus retain most of their radial velocity.

\subsection{Stable Stratification and Overshooting}

As convective motions enter the stable stratification of the radiative envelope, they are buoyantly braked (see $\S 3.2$ ). The amount of braking they experience is controlled by the magnitude of the stable entropy gradient. By varying that gradient in our simulations, we have thus effectively altered the ability of flows to overshoot into the radiative envelope: a larger $d \bar{S} / d r$ implies stronger buoyancy braking and thus a smaller extent to the overshooting region. A larger $d \bar{S} / d r$ also leads to a smaller extent to the penetration as indicated by $r_{c}$. Both these effects are apparent in Figure 13, which displays $F_{e}(r)$ at several latitudes, for three simulations of varying $d \bar{S} / d r$. With increasing stiffness comes decreasing $r_{o}$ and $r_{c}$.

For our stiffest and most complex case E, the latitudeaveraged extent of overshoot, $\bar{d}_{o}$, is $1.906 \times 10^{9} \mathrm{~cm}$, or $0.208 H_{p}$, where $H_{p}$ is the local pressure scale height averaged over the region of overshoot. In the less stiff case $\mathrm{C}$, a larger $\bar{d}_{o}$ is realized; such measures are quoted for all cases in Table 2 . Increasingly complex flows, as achieved in our simulations by lowering the viscosity in going from case $\mathrm{B}$ to case $\mathrm{C}$ (or from case $\mathrm{D}$ to case $\mathrm{E}$, at a different stiffness), yield smaller $\bar{d}_{o}$ than do their more laminar relatives. This seems at first paradoxi$\mathrm{cal}$, given the larger rms velocities associated with the increased complexity of the convection. However, this trend is largely in keeping with nonlinear modeling of three-dimensional penetrative compressible convection in localized planar
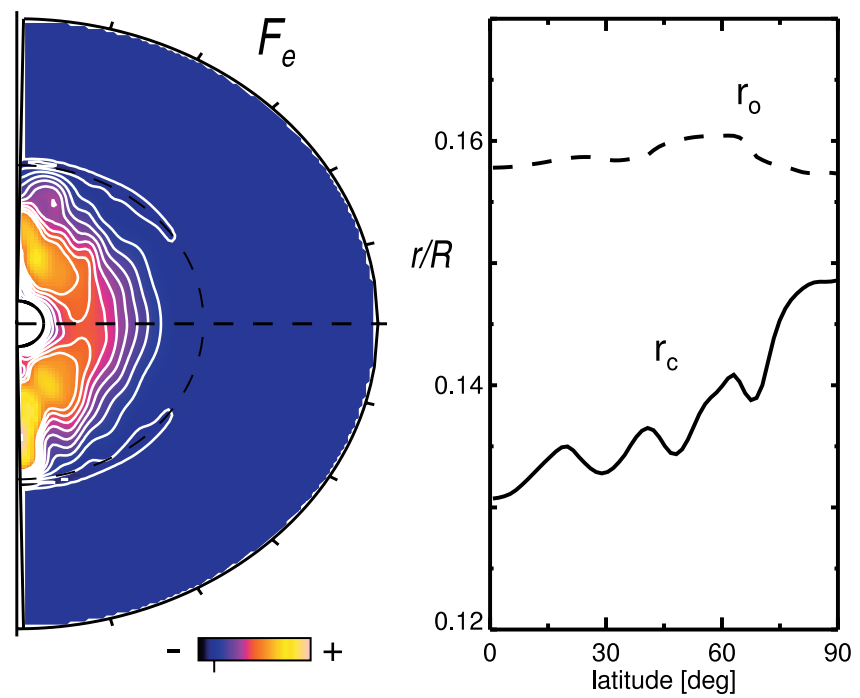

FIG. 11.-(a) Variation with radius and latitude of the radial enthalpy flux $\hat{F}_{e}$ in case $\mathrm{C}$, averaged over 180 days. This reveals the complex patterns to the transport achieved by the vigorous convection within the core (with positive flux denoted in yellow/red tones) and the weaker response in the overshooting region (with a negative flux). The equator is denoted by the dashed line. (b) Assessment of $r_{c}$ and $r_{o}$ for the same case, expressed in proportional radius as a function of latitude. Using $\hat{F}_{e}, r_{c}$ (solid line) measures the radius at the outer edge of the convective core at which the enthalpy flux becomes negative, and $r_{o}$ the radius of overshooting at which that flux essentially vanishes.

domains (see Brummell et al. 2002), which showed that a smaller filling factor for the plumes as realized in the more turbulent convection translates into a smaller extent to the overshooting.

Increasing $\Omega_{0}$ without altering the viscosity leads to more laminar convection, because of the stabilizing effects of rotation. This in turn yields a greater overshooting. In devising case $\mathrm{C} 4$ with its fourfold greater rotation rate, we reduced the viscosity relative to case $\mathrm{C}$ in order to achieve comparable complexity in the convection as measured by the Reynolds numbers. In case $\mathrm{C} 4, \bar{d}_{0}$ is greater than that in the slower

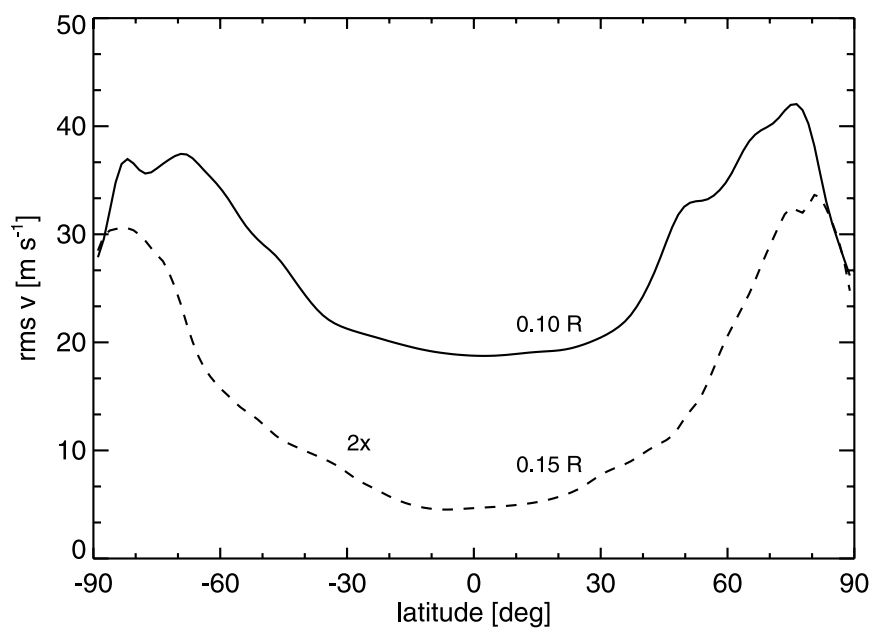

FIG. 12.-Variation with latitude of the rms of radial velocities $\tilde{v}_{r}$ in case C within both the convective core $(r=0.10 R)$ and the region of overshooting $(r=0.15 R)$, averaged over 180 days. The latter velocities have been multiplied by a factor of 2 for visibility. Radial velocities are highest near the poles and lowest near the equator, partly because of the deflection of outward motions by the Coriolis forces in these rotating domains. The prolate geometry of the convective core appears to arise from such latitudinal variation of $v_{r}$. 


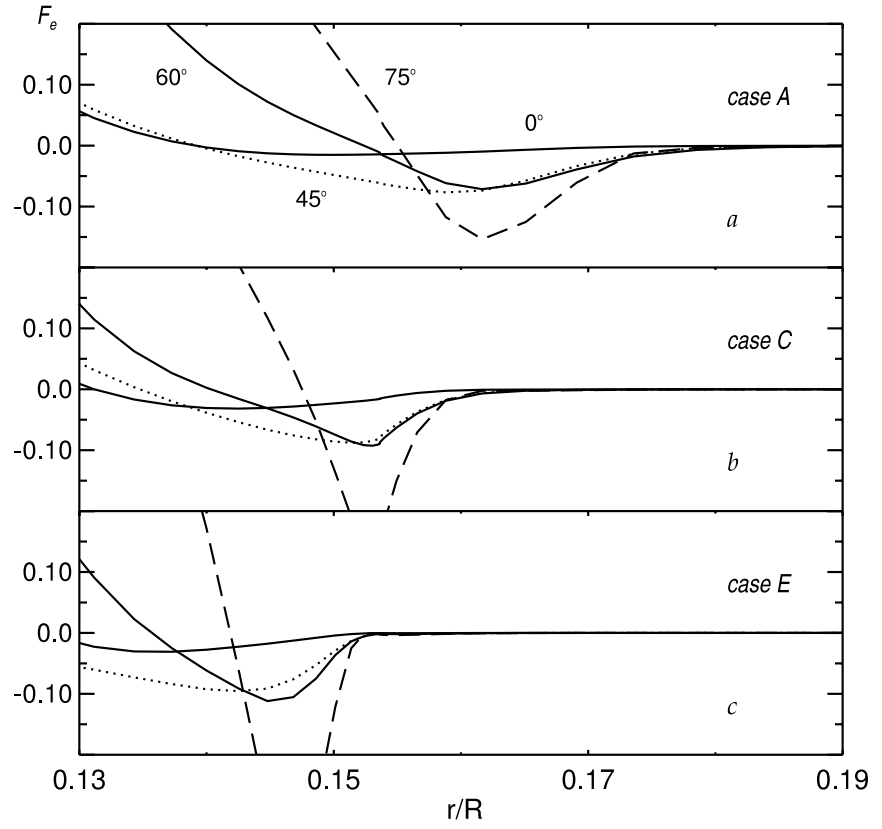

FIG. 13.- Variation of radial enthalpy flux $\hat{F}_{e}$ with radius at selected indicated latitudes for cases $\mathrm{A}, \mathrm{C}$, and $\mathrm{E}$ involving progressively stiffer stable stratifications in the radiative exterior. The radius $r_{c}$ at which the enthalpy flux first becomes negative (providing an estimate of the boundary of the convective core) is a strong function of latitude, with higher latitudes having a core of greater spatial extent. In contrast, the radius $r_{o}$ at which the negative enthalpy flux in the overshooting region essentially vanishes is largely insensitive to latitude. Increasing stiffness decreases the radii at which these transitions occur.

rotating case $C$, suggesting that increasing the rotation rate enhances somewhat the overshooting.

\section{INDUCED MERIDIONAL CIRCULATIONS}

Prominent meridional circulations are induced by the convection within the unstable core, accompanied by much weaker circulations in the radiative surroundings. Within the convective core, such mean meridional flows arise from a combination of buoyancy forces, Reynolds stresses, latitudinal pressure gradients, and Coriolis forces acting on the mean zonal flow (differential rotation). In the radiative exterior beyond the zone of overshooting, slow circulations can be driven both by viscous stresses that couple that region to the flows in the convective interior and by latitudinal temperature gradients arising from the nonuniform heating from below. Our simulations reveal that the resulting meridional circulations are complex in structure, possess multiple cells in both radius and latitude, and exhibit noticeable variations in time. Such multicellular patterns are in sharp contrast to the single monolithic cells often assumed in mean-field models of differential rotation (e.g., Rekowski \& Rüdiger 1998; Durney 2000). On the other hand, our models include viscous stresses that may be largely absent in real stars, and thus our estimates of the meridional circulations in the immediate surroundings of the convective core are likely to be somewhat unrealistic.

\subsection{Patterns within Convective Core}

The time-averaged meridional circulations achieved in three of our simulations are shown in Figure 14 as stream functions of mass flux (see eq. [7] of Miesch et al. 2000). Within the convective core (Fig. 14, left), these flows have amplitudes of order $5 \mathrm{~m} \mathrm{~s}^{-1}$; they are thus appreciably smaller than the instantaneous radial velocities in the same simulations (see Table 2). Indeed, the kinetic energy of these axisymmetric meridional flows (MCKE) is in all cases at least an order of magnitude smaller than that of the nonaxisymmetric convection (CKE) and of the differential rotation (DRKE). More rapid flows and more intricate cellular patterns are realized in the less viscous cases $\mathrm{C}$ and $\mathrm{C} 4$ than in case $\mathrm{B}$.

The patterns of meridional flow are quite intricate, with multicelled structures achieved in radius and latitude. These structures exhibit some alignment with the rotation axis, particularly in the more rapidly rotating case $\mathrm{C} 4$. As the convection itself evolves (Fig. 8), so do the mean meridional circulations associated with it. Thus, Figure 14 represents

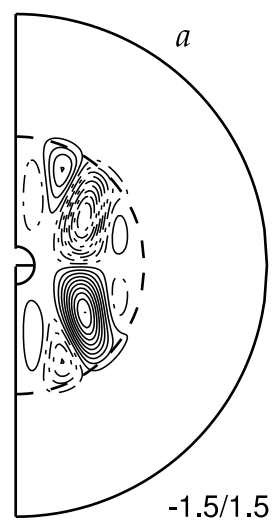

case $B$
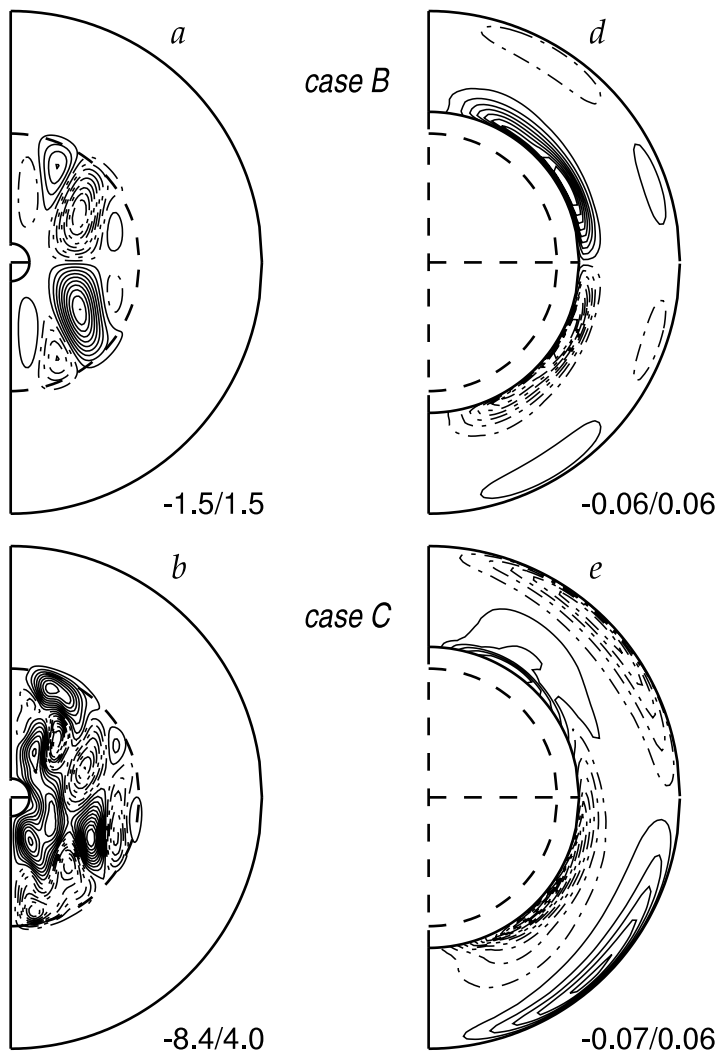

case $C$
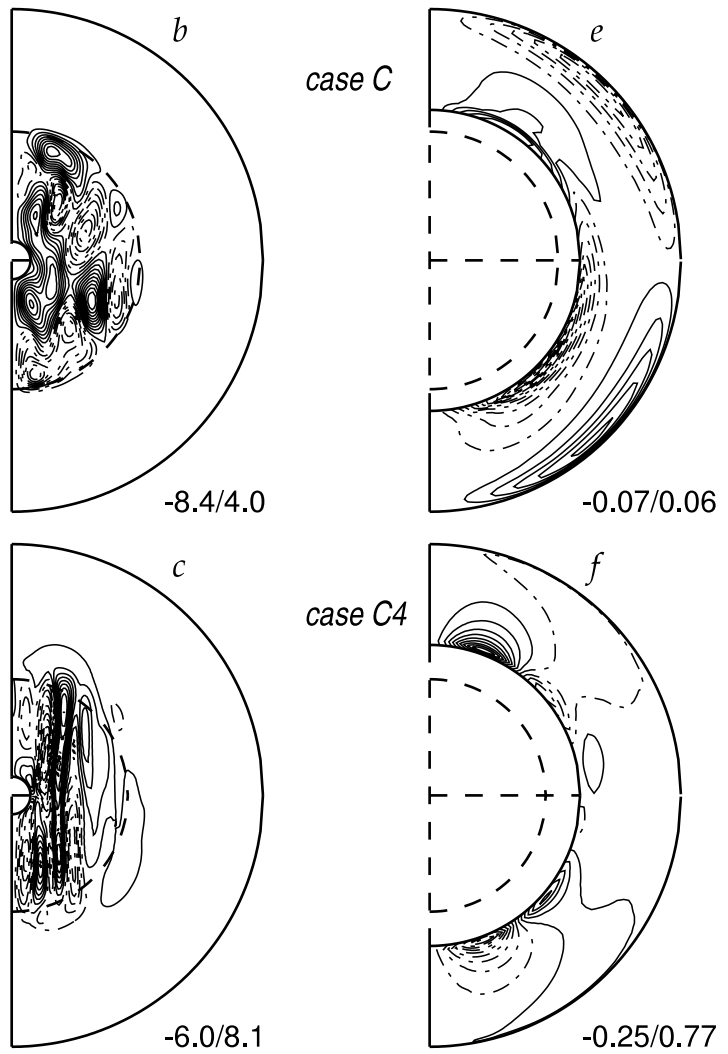

FIG. 14.- Time-averaged stream functions of meridional mass flux for cases B (top), C (middle), and C4 (bottom), displayed for the whole domain (left) and at much amplified scaling for the outer portion of the radiative zone only (right). The time intervals used in the averaging are all of order 110 days. Solid lines denote counterclockwise flow, dashed lines clockwise. The associated maximum and minimum velocities for $\tilde{v}_{\theta}$ (in meters per second) are indicated. 
typical examples of these time-dependent circulations in a particular epoch. These circulations occupy most of the volume delineated by $r_{c}$ and weaken in the region of overshooting. Thus, their extent varies with the stiffness of the exterior stratification.

\subsection{Weak External Circulations}

The meridional circulations achieved in the radiative envelope within our models are much weaker than those in the core. As shown in Figure 14 (right), these flows are of large scale and possess amplitudes that in cases $\mathrm{B}$ and $\mathrm{C}$ are about $0.06 \mathrm{~m} \mathrm{~s}^{-1}$. The more rapidly rotating case $\mathrm{C} 4$ exhibits faster exterior circulations. Such flows evolve more slowly than do the meridional circulations within the core. They are driven by a combination of viscous stresses communicated across these domains (with a characteristic diffusion timescale across the radiative envelope of about $1.8 \mathrm{yr}$ for case $\mathrm{C}$ ) and by weak latitudinal thermal gradients resulting from the energy deposited by the latitudinally varying $\hat{F}_{e}$ (Fig. 11).

These mean flows within the radiative envelopes in our models are considerably greater in amplitude than estimates of Eddington-Sweet circulations. The latter, which arise from baroclinic effects due to the flattened shape of a rotating star, would involve flows of order $10^{-4} \mathrm{~m} \mathrm{~s}^{-1}$ in our A-type stars rotating at $\Omega_{0}$ (see review in Pinsonneault 1997). Our modeling ignores centrifugal acceleration and thus such distortions and further imposes a spherical outer boundary upon which a uniform $d S / d r$ is specified. Therefore, our model does not accurately address the very slow circulations of the Eddington-Sweet variety.

\section{INTERPRETING THE ROTATION PROFILES}

Our simplified model of rotating core convection coupled by penetration to a radiative envelope yields a nonlinear dynamical system with complex feedbacks. The rich variety of responses and marked time variability that is exhibited cannot now be predicted or explained from first principles. However, the numerical simulations have the great advantage that they provide full snapshots that can be assessed for the dynamical balances that are achieved. This can yield insights into the operation of these complicated dynamical systems. Such an approach is particularly useful in identifying the processes involved in continuously redistributing the angular momentum to establish the differential rotation. Because we have adopted stress-free boundary conditions at the top and bottom boundaries of our simulations, no net torque is applied to these rotating spheres of convection. Thus, total angular momentum within our simulations is conserved, and we now examine the manner in which it is redistributed.

\subsection{Redistributing the Angular Momentum}

We may assess the transport mechanisms of angular momentum in our simulations, which must combine to give rise to the observed differential rotation, in the manner of Elliott et al. (2000) and Brun \& Toomre (2002). We consider the mean radial $\left(\mathcal{F}_{r}\right)$ and latitudinal $\left(\mathcal{F}_{\theta}\right)$ angular momentum fluxes and write the $\phi$-component of the momentum equation, expressed in conservative form and averaged in time and longitude, as

$$
\frac{1}{r^{2}} \frac{\partial\left(r^{2} \mathcal{F}_{r}\right)}{\partial r}+\frac{1}{r \sin \theta} \frac{\partial\left(\sin \theta \mathcal{F}_{\theta}\right)}{\partial \theta}=0,
$$

involving the mean radial angular momentum flux

$$
\mathcal{F}_{r}=\hat{\rho} r \sin \theta\left[-\nu r \frac{\partial}{\partial r}\left(\frac{\hat{v}_{\phi}}{r}\right)+\widehat{v_{r}^{\prime} v_{\phi}^{\prime}}+\hat{v}_{r}\left(\hat{v}_{\phi}+\Omega r \sin \theta\right)\right]
$$

and the mean latitudinal angular momentum flux

$$
\begin{aligned}
\mathcal{F}_{\theta}= & \hat{\rho} r \sin \theta\left[-\nu \frac{\sin \theta}{r} \frac{\partial}{\partial \theta}\left(\frac{\hat{v}_{\phi}}{\sin \theta}\right)+\widehat{v_{\theta}^{\prime} v_{\phi}^{\prime}}\right. \\
& \left.+\hat{v}_{\theta}\left(\hat{\boldsymbol{v}}_{\phi}+\Omega r \sin \theta\right)\right] .
\end{aligned}
$$

In equations (13) and (14), the first term in each pair of brackets is related to the angular momentum flux due to viscous transport (which we denote $\mathcal{F}_{r, V}$ and $\mathcal{F}_{\theta, V}$ ), the second term to the transport due to Reynolds stresses $\left(\mathcal{F}_{r, R}\right.$ and $\left.\mathcal{F}_{\theta, R}\right)$, and the third term to the transport by the meridiona circulation $\left(\mathcal{F}_{r, M}\right.$ and $\left.\mathcal{F}_{\theta, M}\right)$. The Reynolds stresses in the above expressions arise through correlations of the components of velocity.

In Figure 15, we show the components of $\mathcal{F}_{r}$ and $\mathcal{F}_{\theta}$ for cases $D$ and $E$ with a strong rotational influence, integrated along colatitude and radius, respectively, to deduce the net fluxes through shells at various radii and through cones at various latitudes, such that

$$
\begin{aligned}
& I_{\mathcal{F}_{r}}(r)=\int_{0}^{\pi} \mathcal{F}_{r}(r, \theta) r^{2} \sin \theta d \theta, \\
& I_{\mathcal{F}_{\theta}}(\theta)=\int_{r_{\text {bot }}}^{r_{\text {top }}} \mathcal{F}_{\theta}(r, \theta) r \sin \theta d r .
\end{aligned}
$$

We have identified the contributions from viscous $(V)$, Reynolds stress $(R)$, and meridional circulation $(M)$ terms. This representation is helpful in considering the sense and amplitude of the transport of angular momentum within the convective shells by each component of $\mathcal{F}_{r}$ and $\mathcal{F}_{\theta}$.

Turning first to the radial fluxes of angular momentum (Figs. $15 a$ and $15 b$ ), we see that viscous forces act to transport angular momentum radially inward in both cases. In this they are opposed by a combination of the Reynolds stress and meridional circulation fluxes, to yield a total radial angular momentum flux that is nearly zero, as noted in Figure 15 by the solid line. While the systems here are highly variable in time, by allowing the system to evolve for extended periods of time (typically thousands of days) and performing long time averages, we appear to be sensing the equilibrated states reasonably well. In going from the more laminar flows of case $D$ to the complex ones of case $E$, we see that the viscous flux has dropped and that the Reynolds stresses and meridional circulations have changed accordingly to maintain equilibrium. This includes the development of an appreciable integrated $\mathcal{F}_{r, M}$ that is directed radially inward, near the boundary of the radiative and convective zones; this is presumably a result of the stronger circulations and shear generated near that boundary by the more complex flows of case $E$.

Examining the latitudinal transport of angular momentum (Figs. $15 c$ and $15 d$ ), we note that the effect of the Reynolds stresses in both cases is primarily to speed up the equator, since $\mathcal{F}_{\theta, R}$ is positive in the northern hemisphere and negative in the southern. It is opposed by the meridional circulation and viscous fluxes, which act to speed up the poles. The manner in which each of the different components of $\mathcal{F}_{\theta}$ acts does not appear to vary appreciably in going from case $D$ to $E$. As the level of complexity is increased, however, we see a decrease in the magnitude of all components of $\mathcal{F}_{\theta}$. 

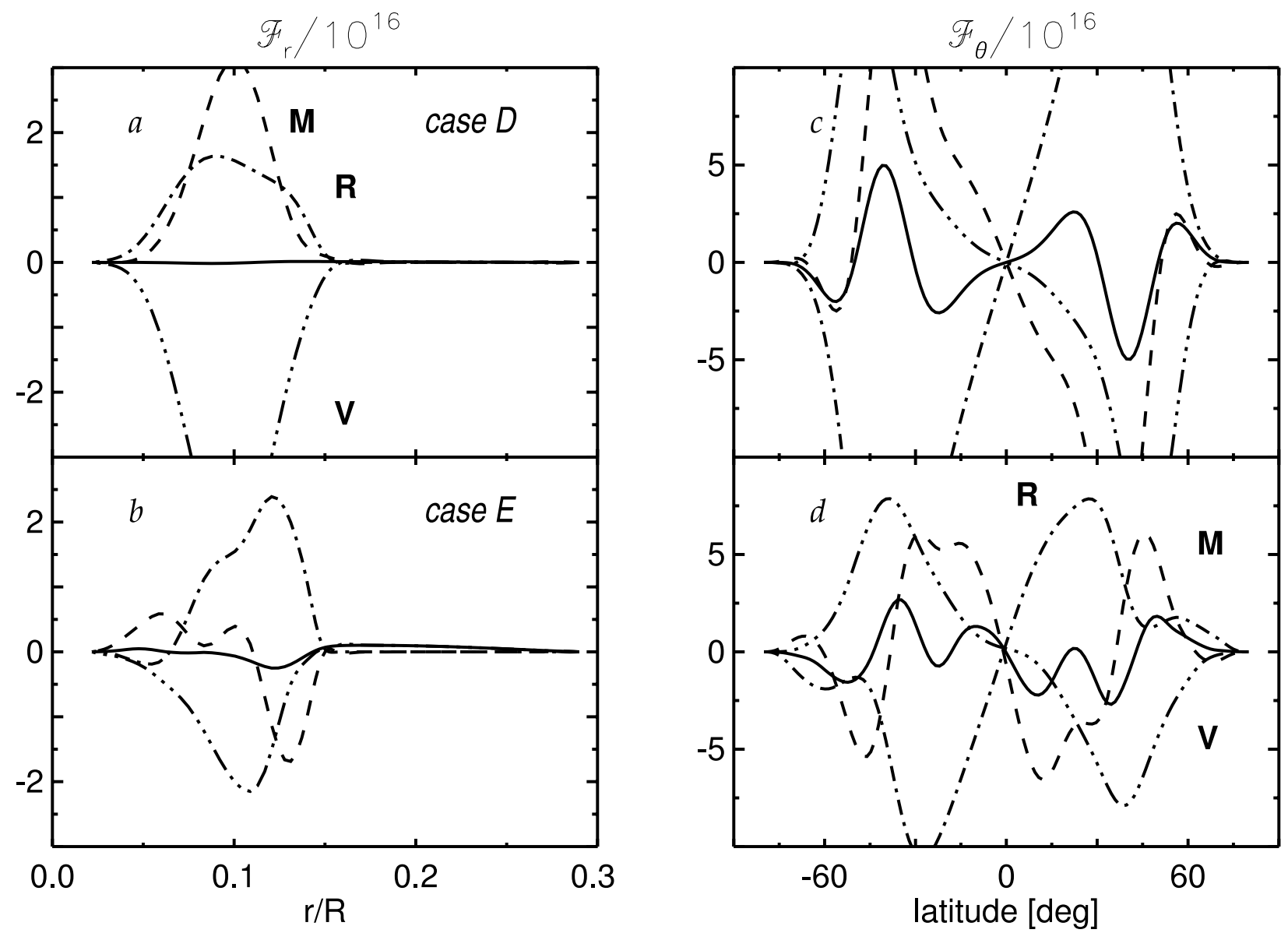

FIG. 15.-Time average of the latitudinal integral of the angular momentum flux $\mathcal{F}_{r}$ (left) and of the radial integral of the angular momentum flux $\mathcal{F}_{\theta}($ right) for

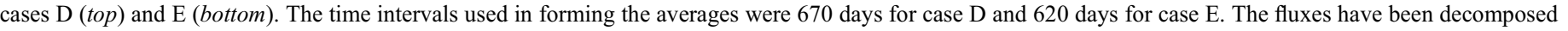

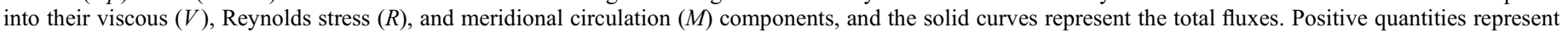
fluxes radially outward or latitudinally from north to south.

We conclude that for these cases involving a strong rotational constraint, the Reynolds stresses act latitudinally to speed up the equator and radially to slow down the convective core. These latitudinal fluxes are opposed by the meridional circulations and viscous effects, whereas the radial fluxes are aided somewhat by the meridional circulations. Examining these fluxes gives us a picture of how the angular momentum is continuously transported and which are the significant processes serving to speed up or slow down certain regions. However, it is difficult to infer from the fluxes alone what will be the detailed $\Omega$ profile that results.

\subsection{Baroclinicity and Thermal Winds}

Rotating convection involves both radial and latitudinal heat transport, with the likelihood that latitudinal gradients in temperature and entropy may result within the convective core. This implies that surfaces of mean pressure and density will not coincide, thereby yielding baroclinic terms in the vorticity equations (Pedlosky 1987; Zahn 1992). Under sufficiently strong rotational constraints, a "thermal wind balance" might be achieved in which departures of the angular velocity from being constant on cylinders (aligned with the rotation axis) are controlled by those baroclinic terms. Indeed, some mean-field approaches have invoked such a balance to obtain differential rotation profiles with bearing on the solar convection zone (e.g., Kichatinov \& Rüdiger 1995). As dis- cussed in Brun \& Toomre (2002), such a balance effectively implies that

$$
\frac{\partial \hat{v}_{\phi}}{\partial z}=\frac{1}{2 \Omega_{0} \hat{\rho} c_{P}} \nabla \hat{S} \times\left.\nabla \hat{p}\right|_{\phi}=\frac{g}{2 \Omega_{0} r c_{P}} \frac{\partial \hat{S}}{\partial \theta}
$$

where $z$ is parallel to the rotation axis. Thus, latitudinal entropy gradients could serve to break the Taylor-Proudman constraint, which would otherwise require the rotation to be constant on cylinders with $\partial \hat{v}_{\phi} / \partial z$ equal to zero. That constraint may also be broken by Reynolds and viscous stresses. We next show that these terms are at least as important as the baroclinic terms in establishing the differential rotation in the bulk of the convective core.

Figure 16 assesses for case $\mathrm{E}$ the extent to which latitudinal entropy gradients serve to drive the temporal mean zonal flows $\hat{v}_{\phi}$ seen as the differential rotation. Figure $16 b$ displays the gradient $\partial \hat{v}_{\phi} / \partial z$ of those zonal flows, and Figure $16 c$ the corresponding latitudinal entropy term on the right-hand side of equation (16), which in an exact thermal wind balance would be identical. Figure $16 d$ shows the difference between this baroclinic term and the actual $\partial \hat{v}_{\phi} / \partial z$, thus providing a measure of departures from such a balance. Within the bulk of the convective core, Reynolds and viscous stresses lead to zonal flows that are not so balanced. At the interface between 

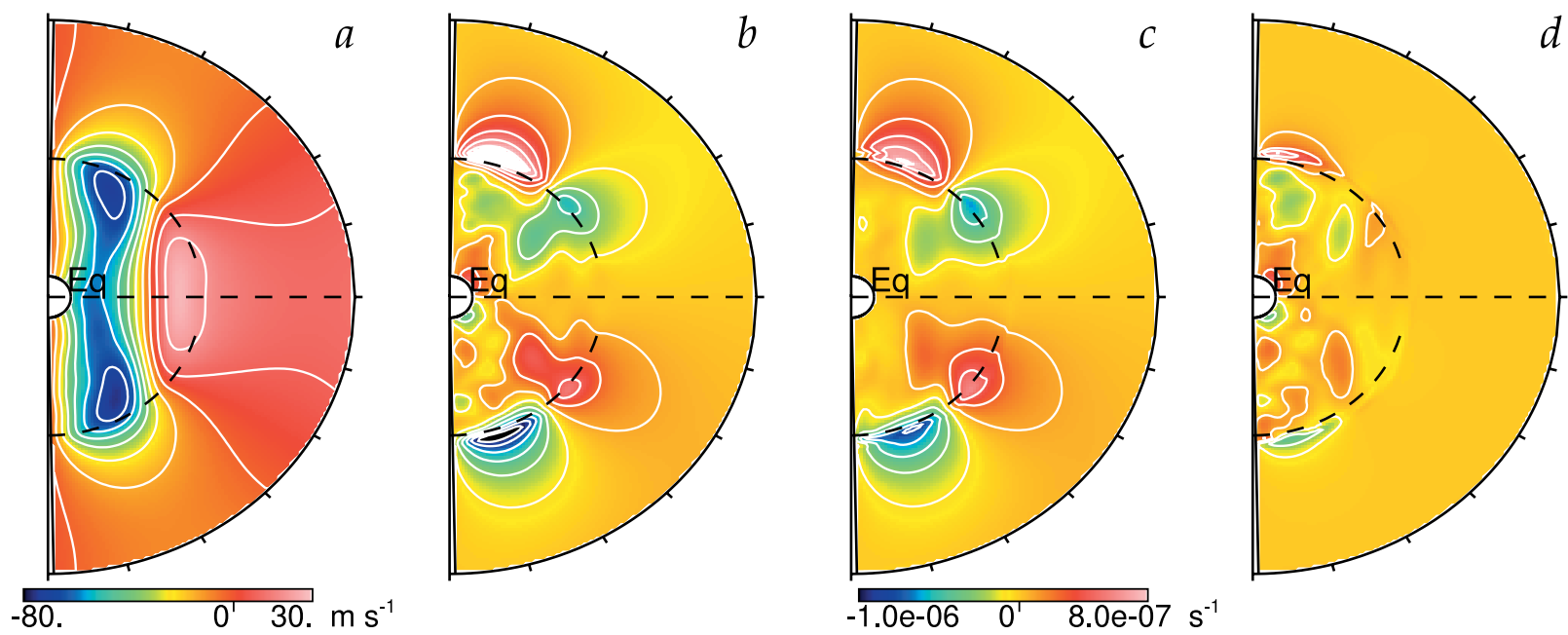

FIG. 16. - Temporal (640 day) and longitudinal averages for case E showing $(a)$ the longitudinal velocity $\hat{v}_{\phi},(b) d \hat{v}_{\phi} / d z,(c)$ the baroclinic term in the meridional

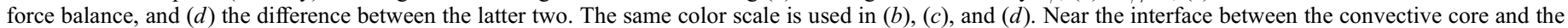

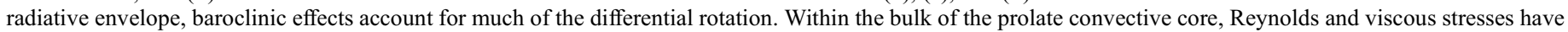
a significant role in driving those zonal flows.

the core and the radiative exterior, however, baroclinicity accounts for most of the differential rotation.

Thus, examining the nature of this thermal wind balance, combined with the assessment of fluxes of angular momentum, reveals that Reynolds and viscous stresses have a major role in establishing the differential rotation in the bulk of the core, with latitudinal thermal gradients influencing the profile near the interface between the convective region and the radiative envelope.

\section{SUMMARY AND PERSPECTIVES}

Our highly simplified treatment of core convection within A-type stars has begun to capture some of the complex dynamics that must be occurring when turbulent convection and rotation meet in a full spherical domain. The most severe simplifications in our models are that we are able to simulate only the central portions of these stars, that compositional gradients that must be present are ignored, and that the levels of turbulence studied here are only modest compared with what may be realized in real stars. The dynamics may well be influenced by each in turn and in ways that are difficult to predict at this stage. Although our work is thus quite preliminary, it has revealed several dynamical properties that may well turn out to be robust.

First, these convective cores rotate differentially. Prominent angular velocity contrasts are established in all cases studied here, with a central column of slow rotation realized in stars that are strongly influenced by rotation (with $R_{o c}$ small). Within the bulk of the convection zone, this differential rotation is driven primarily by Reynolds and viscous stresses, whereas near the interface between the core and the radiative envelope, baroclinicity plays an important role.

Second, convective motions penetrate into the radiative envelope, yielding a prolate shape to the region of nearly adiabatic stratification. The surrounding region of overshooting, in which convective motions persist but do not appreciably modify the prevailing entropy gradient, is found to have an outer boundary that is largely independent of latitude, yielding a spherical shape to the domain experiencing convective stirring.
Third, small-amplitude circulations and gravity waves are excited within the radiative envelope. The latter appear in some simulations as a rippling patchwork of waves and in our more rapidly rotating case $\mathrm{C} 4$ as a striking global-scale resonance that, once established, appears to persist.

The simplifications we have made in our modeling are likely to impact the penetrative properties to some degree. In particular, the $\mu$-gradients of chemical composition present in real stars should diminish the extent of penetrative and overshooting motions realized there (Zahn 1991). They also probably reduce the vigor of circulations within the radiative envelope (Lebreton \& Maeder 1987). More turbulent flows are likely to yield smaller spatial filling factors for the convective plumes and hence probably also lessen the extent of overshooting (Brummell et al. 2002). Furthermore, real stars possess stiffer entropy stratifications in their radiative envelopes than those we have been able to consider. This too would lead to less overshooting. Thus, our estimates here provide upper limits for the extent of penetration and overshooting that may be achieved in real stars.

The strong regions of shear realized in all our simulations may contribute to the building of orderly magnetic fields by dynamo action. Poloidal fields can be converted into toroidal ones by such differential rotation (the $\Omega$-effect), with regeneration of the poloidal fields perhaps accomplished by the helical turbulence (the $\alpha$-effect; see Mestel 1999). Acting together, these processes may build well-organized fields that could, if subject to magnetic buoyancy instabilities, rise to the surfaces of these stars, where they might be observed (MacGregor \& Cassinelli 2003). We are in the process of beginning to explore the generation of magnetic fields within these cores (see Browning, Brun, \& Toomre 2004).

The penetrative convection and overshooting in our simulation may likewise have some observable consequences. Although we have not investigated in any detail the bringing of fresh fuel into the convective core by these motions, some replenishment of the hydrogen stockpile available for nuclear reactions must occur and thereby lead to a prolonged lifetime on the main sequence. 
We thank Douglas Gough and Jean-Paul Zahn for very helpful discussions. J. T. thanks the Observatory of Paris, Meudon, and the CEA-Saclay for their hospitality during the interpretation of these simulations. This work was partly supported by NASA through SEC Theory Program grant NAG511879 and through the Graduate Student Researchers Program
(NGT5-50416). Various phases of the simulations here were carried out with NSF PACI support of the San Diego Supercomputing Center, the National Center for Supercomputing Applications, and the Pittsburgh Supercomputing Center. Much of the analyses of the extensive data sets were conducted in the Laboratory for Computational Dynamics within JILA.
Abt, H. A. 2000, ApJ, 544, 933

Adelberger, E., et al. 1998, Rev. Mod. Phys., 70(4), 1265

Browning, M., Brun, A. S., \& Toomre, J. 2004, in Proc. IAU Symp. 215, Stellar Rotation, ed. A. Maeder \& P. Eenens (San Francisco: ASP), in press Brummell, N. H., Clune, T. L., \& Toomre, J. 2002, ApJ, 570, 825

Brummell, N. H., Hurlburt, N. E., \& Toomre, J. 1998, ApJ, 493, 955

Brun, A. S., Antia, H. M., Chitre, S. M., \& Zahn, J.-P. 2002, A\&A, 391, 725

Brun, A. S., \& Toomre, J. 2002, ApJ, 570, 865

Carraro, G., Chiosi, C., Bressan, A., \& Bertelli, G. 1994, A\&AS, 103, 375

Chandrasekhar, S. 1961, Hydrodynamic and Hydromagnetic Stability (Oxford: Clarendon)

Charbonneau, P., \& MacGregor, K. B. 2001, ApJ, 559, 1094

Clune, T. L., Elliott, J. R., Glatzmaier, G. A., Miesch, M. S., \& Toomre, J. 1999, Parallel Comput., 25, 361

Cunha, M. S. 2002, in Radial and Nonradial Pulsations as Probes of Stellar Physics, ed. C. Aerts, T. R. Bedding, \& J. Christensen-Dalsgaard (San Francisco: ASP), 272

Dintrans, B., \& Rieutord, M., \& Valdettaro, L. 1999, J. Fluid Mech., 398, 271

Durney, B. R. 2000, ApJ, 528, 486

Elliott, J. R., Miesch, M. S., \& Toomre, J. 2000, ApJ, 533, 546

Gehren, T. 1988, Rev. Mod. Astron., 1, 52

Gilman, P. A. 1978, Geophys. Astrophys. Fluid Dyn., 11, 157 1979, ApJ, 231, 284

Gough, D. O. 1993, in Astrophysical Fluid Dynamics, ed. J.-P. Zahn \& J. ZinnJustin (New York: North-Holland), 399

Gough, D. O., \& Toomre, J. 1991, ARA\&A, 29, 627

Greenspan, H. P. 1969, The Theory of Rotating Fluids (Cambridge: Cambridge Univ. Press)

Hurlburt, N. E., Toomre, J., Massaguer, J. M., \& Zahn, J.-P. 1994, ApJ, 421, 245

Iglesias, C., \& Rogers, F. J. 1996, ApJ, 464, 943

Kichatinov, L. L., \& Rüdiger, G. 1995, A\&A, 299, 446

Kurtz, D. W. 1990, ARA\&A, 28, 607

Latour, J., Toomre, J., \& Zahn, J.-P. 1981, ApJ, 248, 1081

Lebreton, Y., \& Maeder, A. 1987, A\&A, 175, 99

MacGregor, K. B., \& Cassinelli, J. P. 2003, ApJ, 586, 480

Maeder, A., \& Meynet, G. 1991, A\&AS, 89, 451

\section{EFERENCES}

Maeder, A., \& Meynet, G. 2000, ARA\&A, 38, 143

Matthews, J. 1991, PASP, 103, 5

Mestel, L. 1999, Stellar Magnetism (Oxford: Clarendon)

Meynet, G., Mermilliod, J.-C., \& Maeder, A. 1993, A\&AS, 98, 477 Michaud, G., Tarasick, D., Charland, Y., \& Pelletier, C. 1983, ApJ, 269, 239

Miesch, M. S., Elliott, J. R., Toomre, J., Clune, T. L., Glatzmaier, G. A., \& Gilman, P. A. 2000, ApJ, 532, 593

Mohanty, S., Basri, G., Shu, F., Allard, F., \& Chabrier, G. 2002, ApJ, 571, 469 Morel, P. 1997, A\&AS, 124, 597

Noyes, R. W., Hartmann, L. W., Baliunas, S. L., Duncan, D. K., \& Vaughan, A. H. 1984, ApJ, 279, 763

Pedlosky, J. 1987, Geophysical Fluid Dynamics (New York: Springer)

Perryman, M. A. C., et al. 1998, A\&A, 331, 81

Pinsonneault, M. 1997, ARA\&A, 35, 557

Preston, G. W. 1974, ARA\&A, 12, 257

Rekowski, B. V., \& Rüdiger, G. 1998, A\&A, 335, 679

Richard, O., Michaud, G., \& Richer, J. 2001, ApJ, 558, 377

Richer, J., Michaud, G., \& Turcotte, S. 2000, ApJ, 529, 338

Rogers, F. J., Swenson, J., \& Iglesias, C. 1996, ApJ, 456, 902

Rosvick, J. M., \& VandenBerg, D. A. 1998, AJ, 115, 1516

Roxburgh, I. W. 1965, MNRAS, 130, 223

. 1978, A\&A, 65, 281

. 1989, A\&A, 211, 361

1992, A\&A, 266, 291

1998, Ap\&SS, 261, 43

Schou, J., et al. 1998, ApJ, 505, 390

Thompson, M., et al. 2003, ARA\&A, 41, 599

Unno, W., Osaki, Y., Ando, H., \& Shibahashi, H. 1979, Nonradial Oscillations of Stars (Tokyo: Univ. Tokyo Press)

Vauclair, G., Vauclair, S., \& Pamjatnikh, A. 1974, A\&A, 31, 63

Vauclair, S. 2003, Ap\&SS, 284, 205

Wolff, S. C. 1983, The A-Stars: Problems and Perspectives (Washington: NASA)

Woo, J.-H., \& Demarque, P. 2001, AJ, 122, 1602

Zahn, J.-P. 1991, A\&A, 252, 179

. 1992, A\&A, 265, 115 